\title{
Review
}

Youngbum Kim and Jeongyong Kim*

\section{Near-field optical imaging and spectroscopy of 2D-TMDs}

https://doi.org/10.1515/nanoph-2021-0383

Received July 18, 2021; accepted September 13, 2021; published online September 29, 2021

\begin{abstract}
Two-dimensional transition metal dichalcogenides (2D-TMDs) are atomically thin semiconductors with a direct bandgap in monolayer thickness, providing ideal platforms for the development of exciton-based optoelectronic devices. Extensive studies on the spectral characteristics of exciton emission have been performed, but spatially resolved optical studies of 2D-TMDs are also critically important because of large variations in the spatial profiles of exciton emissions due to local defects and charge distributions that are intrinsically nonuniform. Because the spatial resolution of conventional optical microscopy and spectroscopy is fundamentally limited by diffraction, near-field optical imaging using apertured or metallic probes has been used to spectrally map the nanoscale profiles of exciton emissions and to study the effects of nanosize local defects and carrier distribution. While these unique approaches have been frequently used, revealing information on the exciton dynamics of 2D-TMDs that is not normally accessible by conventional far-field spectroscopy, a dedicated review of near-field imaging and spectroscopy studies on 2D-TMDs is not available. This review is intended to provide an overview of the current status of near-field optical research on 2D-TMDs and the future direction with regard to developing nanoscale optical imaging and spectroscopy to investigate the exciton characteristics of 2D-TMDs.
\end{abstract}

Keywords: exciton; TEPL; TERS; TMD; two-dimensional materials.

*Corresponding author: Jeongyong Kim, Department of Energy Science, Sungkyunkwan University, Suwon 16419, Republic of Korea, E-mail: j.kim@skku.edu. https://orcid.org/0000-0003-4679-0370 Youngbum Kim, Department of Energy Science, Sungkyunkwan University, Suwon 16419, Republic of Korea; and Center for Integrated Nanostructure Physics, Institute for Basic Science, Suwon 16419, Republic of Korea

\section{Introduction}

Since the discovery of graphene, two-dimensional (2D) materials have been the focus of extensive studies; however, optical applications of graphene and other metallic 2D materials have been limited due to the absence of a bandgap [1-5]. Transition metal dichalcogenides (TMDs) are semiconducting 2D materials with bandgaps that cover the visible and infrared ranges, and the number of layers can be controlled owing to the weak van der Waals interlayer interaction [6]. In addition, the effects of quantum confinement and low dielectric screening cause the binding energies of excitons in 2D-TMDs in a single layer to be very large, i.e., up to a few $100 \mathrm{meV}[7,8]$. This has led to extensive research on various exciton-based optoelectronic applications [9-12].

Atomic force microscopy (AFM), scanning tunneling microscopy (STM), and transmission electron microscopy (TEM) can reveal structural properties such as surface roughness, height, defects, and lattice structure of 2D-TMDs with atomic resolution [13-15]. However, these microscopy techniques do not provide optical responses such as photoluminescence (PL), Raman scattering, and absorption spectra. Confocal optical microscopy facilitates the visualization of the spatial distribution of PL and Raman scattering at diffraction-limited spatial resolution and has been extensively used in the characterization of nanomaterials and devices [16-19]. However, the spatial resolution of confocal microscopy is limited to $\sim 300 \mathrm{~nm}$ $[20,21]$, and the near-field scanning microscope is a good option for measuring the optical properties of 2D-TMDs [22-25] with a spatial resolution higher than $100 \mathrm{~nm}$. Specifically, near-field scanning microscopy (NSOM), tipenhanced PL (TEPL), and Raman spectroscopy (TERS) techniques have been highly effective in obtaining nanoscale optical images of 2D-TMDs [26-28].

To date, there have been a number of review papers on the use of near-field microscopy on low-dimensional materials [29-33], but no review has been dedicated to near-field optical studies of 2D-TMDs that has brought significant advances on understanding of local optical properties of 2D-TMDs. For instances, ultra-high spatial 
resolution of near-field optical imaging and spectroscopy have enabled the optical identification of nanoscale structural defects such as localized defects, grain boundaries, and wrinkles on 2D-TMDs, and visualized fine modulation of spatial distribution of local charge carriers [26, 34, 35], which are not normally probable by far-field optical imaging. Furthermore, in near-field systems using metallic probes, the optical responses of 2D-TMDs can be manipulated by locally tuning the strain or plasmon coupling $[28,36]$. In this review, studies on the optical properties of 2D-TMDs performed by near-field microscopy are reviewed in terms of the specific interests of the studies such as the identification of defects and carrier densities or the effects of strain and plasmon resonances on the spectral and spatial characteristics of exciton emissions of 2D-TMDs.

\section{Overview of development of near- field optical microscopes}

The resolution of a conventional optical microscope is limited to the Rayleigh criterion given as $0.61 \times \lambda / \mathrm{NA}$, where $\lambda$ is the wavelength of light and NA is the numerical aperture of the lens. A confocal optical microscope integrated with a laser source and detection pinhole can provide a slightly better resolution; however, the spatial resolution is still limited by diffraction [17, 37, 38].

Near-field optical microscopy was developed to overcome the diffraction limit. In 1928, Synge proposed the concept of near-field optical microscopy using a nanoscale hole, where the spatial resolution was limited by the hole size rather than the diffraction or the wavelength of the light [39]. In his paper, Synge suggested that the subwavelength hole must be very close to the object in the "near-field" regime to facilitate the evanescence field. Ash and Nicholls demonstrated Synge's proposal using microwaves in 1972 [40]. Lewis and Pohl first described an optical near-field application in 1984 [41, 42]. Betzig first designed aperture-type near-field scanning optical microscopy (a-NSOM) using a metallized pipette tip with an aperture size of $\sim 100 \mathrm{~nm}$ and achieved a spatial resolution of $\sim \lambda / 43$ $[43,44]$.

Another type of NSOM system designed by Keilmann is a scattering-type scanning near-field optical microscopy (s-SNOM) [45], where an apertureless tip instead of an aperture probe was used to locally scatter the light and the spatial resolution of s-SNOM was determined mostly by the apex size tip. Recently, the concepts of TERS and TEPL were developed by integrating s-SNOM with a plasmonic tip (AFM or STM tip made or coated with Au or Ag) to scatter the near-field signal, and a high spatial resolution beyond the diffraction limit was achieved [27, 36]. Near-field optical microscopes can be categorized into two types: aperture and scattering. Figure 1 shows the schematics of the working principles of the confocal microscope, a-NSOM and s-SNOM.

\section{Identification of exciton profiles around structural defects}

\subsection{Exciton emissions affected by defect formation}

TMDs are composed of the chemical formula $\mathrm{MX}_{2}$, where $\mathrm{M}$ is a transition metal and $\mathrm{X}$ is a chalcogen atom and one transition metal is vertically sandwiched by two chalcogenide atoms [46]. 1L-TMDs display extremely stable formations of bound excitons consisting of electrons and holes even at room temperature with a large exciton binding energy owing to the strong Coulomb interaction, suggesting many promising exciton devices [7, 8]. Depending on the growth methods or physical parameters such as local structural defects and carrier concentration of TMDs, 1L-TMDs show spatially different exciton distributions. Various exciton species such as excitons (bright or dark), trions (positive or negative), and biexcitons (coupled with two exciton) and defect-state excitons lead to complex optical responses [6, 47-49]. Although the structural properties of 1L-TMDs have been characterized by AFM, STM, and TEM [13-15], in order to investigate the variations in the optical responses such as PL and Raman scattering

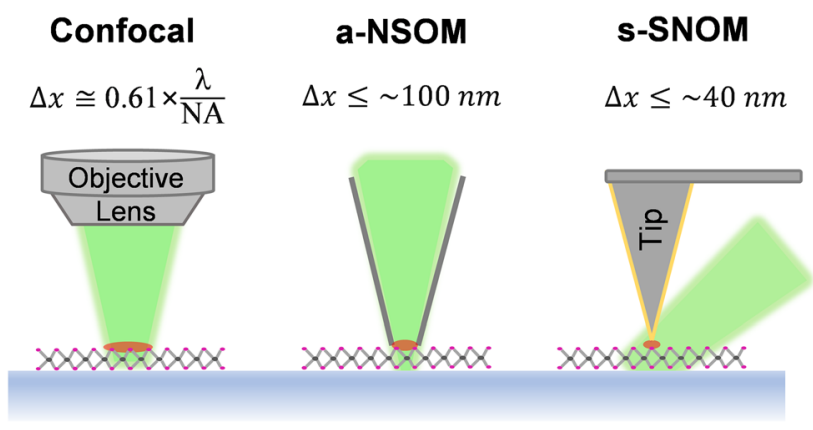

Figure 1: Schematics of confocal, a-NSOM, and S-SNOM microscopes applied to optical investigation of 2D-TMDs. a-NSOM: aperture-type near-field scanning optical microscopy. s-SNOM: scattering-type scanning near-field optical microscopy. $\Delta x$ represents typical size of spatial resolution achievable by respective methods. $\lambda$ : light wavelength; NA: numerical aperture of objective lens. Red spots represent excitation area of 2D-TMDs and green shadow represents laser illumination. 
affected by variations in structural properties, one needs optical tools with a high optical resolution.

Because grain boundaries (GBs) and local defects of 1L-TMDs generated during the growth process with nanoscale dimensions are responsible for the spatial nonuniformity of 1L-TMDs [14, 50], optical investigations using far-field optical microscopy provide limited understanding of the local behavior of exciton complexes in nonuniform $1 \mathrm{~L}$-TMDs requiring the use of near-field imaging combined with PL or Raman spectroscopy. In this section, we focus on the characterization of $1 \mathrm{~L}-\mathrm{TMDs}$ through near-field imaging using NSOM, TERS, or TEPL for nanoscale profiling of exciton emissions of 1L-TMDs.

\subsection{Grain boundary}

PL variation in GBs of 2D-TMDs has been reported to occur as either a PL reduction or increase [50-52]. Nearfield PL images clearly showed the PL variation around the GBs and structural defects such as add-layer and line defects [26]. Figure 2a shows confocal and near-field images of CVD-grown $1 \mathrm{~L}-\mathrm{MoS}_{2}$ with spatial resolutions of $\sim 400$ and $\sim 100 \mathrm{~nm}$, respectively. Unlike the confocal PL image, the near-field PL image clearly shows distinguishable GBs. In addition, the reduction in PL intensity due to physical damage is consistent with previous data [53, 54]. Similar work on PL imaging around GBs was performed by Bao et al. [55], who investigated the nearfield spectral characteristics of CVD-grown $1 \mathrm{~L}-\mathrm{MoS}_{2}$ using a campanile tip, an apertureless-type tip that produced $\sim 60 \mathrm{~nm}$ spatial resolution, as shown in Figure 2b. From near-field spectral imaging, the PL emission of the CVD-grown $1 \mathrm{~L}-\mathrm{MoS}_{2}$ was found to consist of excitons $(1.84 \mathrm{eV})$ and trions $(1.81 \mathrm{eV})$ [56, 57], and PL intensity quenching was observed at the edges and GB regions. This can be explained by the n-doping effect near the edge, and GBs coming from the sulfur vacancies tend to reduce the PL intensity $[58,59]$.

By contrast, in the case of $1 \mathrm{~L}-\mathrm{MoSe}_{2}$, the PL intensity was stronger at the GBs and edge sites, as shown in Figure 2d, which was conducted by TEPL [60]. Because tipinduced plasmon enhancement can generate excess carriers in $1 \mathrm{~L}-\mathrm{MoSe}_{2}$ and cause the doping effect by oxidation, the exciton species of $1 \mathrm{~L}-\mathrm{MoSe}_{2}$ are mostly biexcitons and trions, as shown in Figure 2c, which is consistent with the previous result $[28,61]$. Near-field PL images of trions and biexcitons show that the GBs and edge sites exhibit stronger PL signals both by trions and biexcitons than in other regions. This was attributed to the electron tunneling occurring on GBs and edges owing to the lower dielectric constant and the plasmon coupling [62]. Smithe et al. also performed TERS of CVD-grown $1 \mathrm{~L}-\mathrm{MoSe}_{2}$, as shown in Figure 2e [63].

Figure 2e shows the TERS image and three representative Raman spectra obtained at the resonance (orange), nonresonance (purple) excitation, and GB regions (black). Raman spectra indicate that the $\mathrm{A}_{1}{ }^{\prime}$ mode $\left(240 \mathrm{~cm}^{-1}\right)$ is strong at the nonresonant excitation, and the $\mathrm{E}^{\prime}$ mode $\left(287 \mathrm{~cm}^{-1}\right)$ and at 340,460 , and $550 \mathrm{~cm}^{-1}$ are strong at the
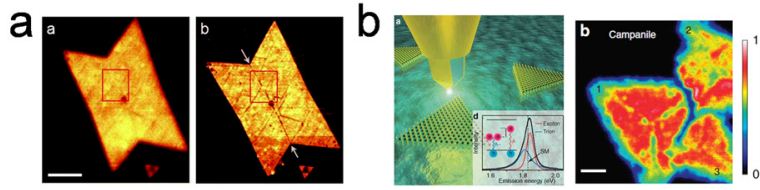

C

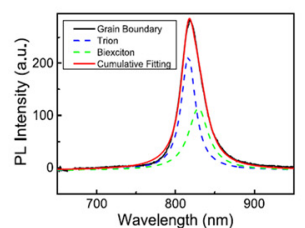

d
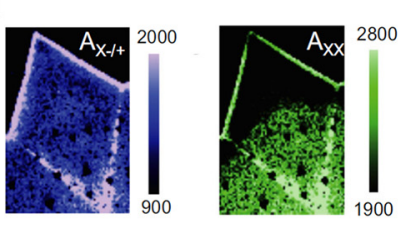
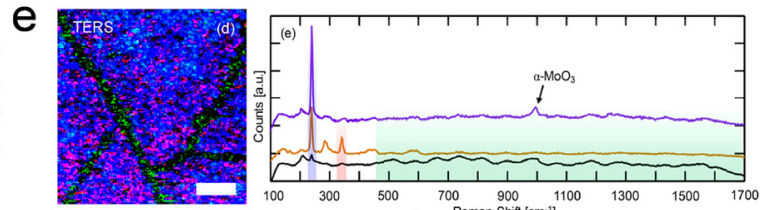

9
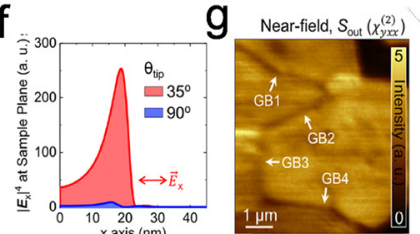

$\mathrm{h}$

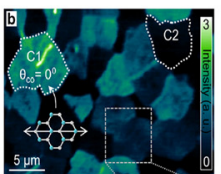

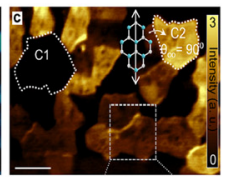

Figure 2: Near-field images of 1L-TMDs.

(a) Confocal (left panel) and near-field (right panel) PL images of 1L-MoS . Reproduced with permission from ref. [26]. Copyright 2015, Royal Society of Chemistry. (b) Illustration (left panel) and near-field PL image (right panel) of $1 \mathrm{~L}-\mathrm{MoS}_{2}$ using the Campanile tip. Inset: representative deconvoluted averaged spectra obtained by near-field image. Reproduced with permission from ref. [55]. Copyright 2015, Nature Publishing Group. (c) Representative deconvoluted averaged PL spectrum of $1 \mathrm{~L}-\mathrm{MoSe}_{2}$. (d) Near-field PL images of trion (left panel) and biexciton (right panel). Reproduced with permission from ref. [60]. Copyright 2020, Nature Publishing Group. (e) TERS image (left panel) and averaged TERS spectra (right panel). Reproduced with permission from ref. [63]. Copyright 2018, American Chemical Society. (f) Simulated optical field profile at sample plane for tilted $\left(\theta_{\text {tip }}=35^{\circ}\right)$ and $\left(\theta_{\text {tip }}=90^{\circ}\right)$ tip orientations. (g) Near-field second harmonic generation (SHG) image. (h) Far-field SHG images measured parallel and perpendicular to the excitation direction configuration. Reproduced with permission from ref. [69]. Copyright 2018, American Chemical Society. 
resonant excitation [64]. In particular, the Raman spectrum near $995 \mathrm{~cm}^{-1}$, known as the out-of-plane molybdenyl bond $\left(\alpha-\mathrm{MoO}_{3}\right)$, was observed using TERS measurements [64, 65]. The nanoscale domain of $\mathrm{MoO}_{3}$ was created during growth. The TERS image shows only the background signal at the GB regions owing to the lack of $\mathrm{MoSe}_{2}$ flakes in the GBs. In general, second harmonic generation (SHG) can identify structural features such as defects, crystal orientation, and stacked angle for bilayers through the nonlinear optical response [66-68]. Park et al. performed SHG near-field imaging of $1 \mathrm{~L}-\mathrm{MoS}_{2}$ with control of the tip orientation [69]. Far-field SHG images showed the typical change in SHG intensity depending on the laser polarization and the orientations of $\mathrm{MoS}_{2}$ grains, as shown in Figure $2 \mathrm{~h}$ [68]. By contrast, the near-field SHG image obtained with $35^{\circ}$ tilted tip showed the uniform SHG contrast regardless of the $\mathrm{MOS}_{2}$ grain orientation, as shown in Figure $2 \mathrm{~g}$. It is because the near-field tip with tilted configuration $\left(\theta_{\text {tip }}=35^{\circ}\right)$ produces both the out-of-plane and in-plane optical fields unlike the normal $\left(\theta_{\text {tip }}=90^{\circ}\right)$ tip that preferentially produces the out-of-plane fields $[26,55$, $60,63]$, whereas far-field SHG imaging uses mostly the inplane illumination that are the most efficient to induce SHG signal [68]. It was observed that near-field SHG intensity obtained at a $35^{\circ}$ tilted tip was 10 times higher than at $90^{\circ}$ normal tip, as shown in Figure 2f.

\subsection{Local defects}

Local defects in 2D-TMDs occur for several reasons. For example, structural defects such as atomic vacancies and line defects such as wrinkles can be created under fluctuating growth conditions or transfer processes $[14,59,70$, 71]. Local defects cause 2D-TMD to be spatially nonuniform and may reduce the quantum yield (QY), which can significantly affect the efficiency of optoelectronic devices. Therefore, precise near-field imaging with a high spatial resolution has been used to determine the roles of local defects. In this section, we focus on the near-field imaging of local defects in 1L-TMDs to study the relationship between optical responses and local defects.

Lee et al. performed near-field imaging of CVD-grown $1 \mathrm{~L}-\mathrm{WS}_{2}$ using an aperture type with $\sim 70 \mathrm{~nm}$ spatial resolution [34]. Figure $3 a$ displays the near-field excitons, trions, and defect-bounded exciton profile images. In this study, researchers observed the presence of localized defect state excitons at room temperature, which were not identified by confocal microscopy owing to the large detection volume. In addition, they found that exciton emission spreads uniformly along the line defects, while trion emission is reduced there. This can be explained by the smaller population of excess electrons on the wrinkles or the different behaviors of diffusion of excitons and trions. Defect-bounded excitons were mostly detected around the line defects, which is consistent with the nature of defect-bound excitons [72, 73].

On the other hand, TERS imaging of 1L-TMDs has also provided spatial information about local defects in 1L-TMDs, which is not usually detected by far-field optical microscopy. TERS images with high spatial resolution and enhanced Raman signals identified the local defect regions [74-76]. In particular, Lee et al. first observed defectrelated Raman modes from CVD-grown $1 \mathrm{~L}-\mathrm{WS}_{2}$ at room a
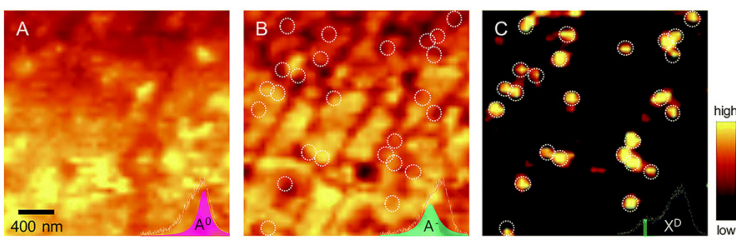

b

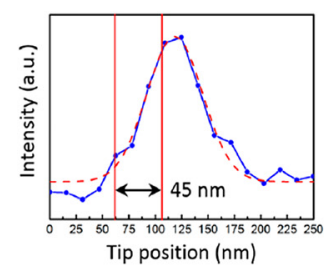

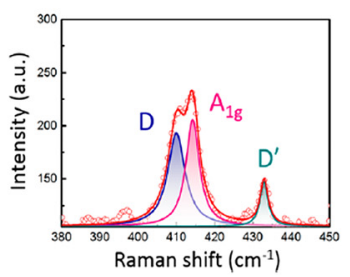
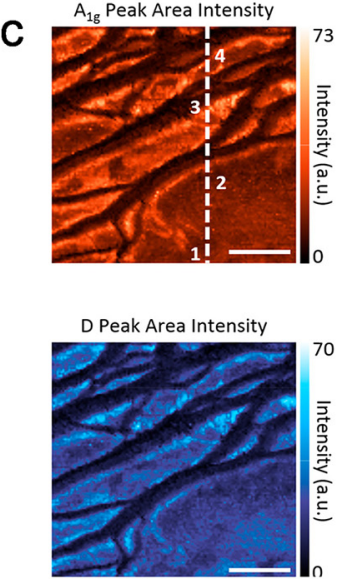
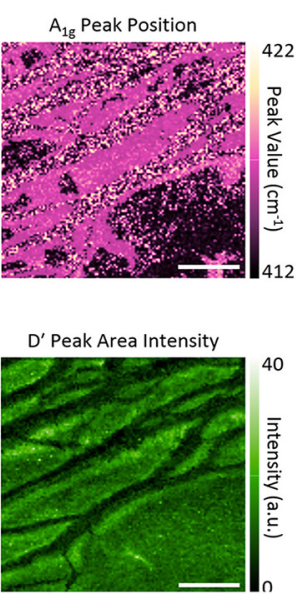

Figure 3: Near-field images of CVD grown 1L-TMDs.

(a) Near-field PL images of exciton, trion, and local defect exciton of 1L-WS 2 , respectively. Reproduced with permission from ref. [34]. Copyright 2017, Royal Society of Chemistry. (b) Line profile of TERS intensity (upper panel) and TERS spectrum of 1L-WS 2 (lower panel). (c) TERS images of $A_{1 g}$ peak area intensity and peak position, respectively (upper panel) and D peak and $D^{\prime}$ intensity, respectively (lower panel). Reproduced with permission from ref. [27]. Copyright 2018, American Chemical Society. 
temperature using a TERS system; the results are shown in Figure $3 \mathrm{c}$ and $\mathrm{d}$ [27]. Owing to the plasmonic enhancement occurring between the tip and substrate and the $\sim 45 \mathrm{~nm}$ spatial resolution, defect-related Raman modes D and D peaks, referred to as $B_{1 u}$, were observed only in the bilayer $\mathrm{WS}_{2}$, as shown in Figure 3c [77, 78]. Figure 3d shows the separated near-field TERS peak position images of the $A_{1 g}$, $\mathrm{D}$, and $\mathrm{D}^{\prime}$ modes and the intensity of the $\mathrm{A}_{1 \mathrm{~g}}$ mode. The intensities of the $\mathrm{D}$ and $\mathrm{D}^{\prime}$ modes are spatially correlated with the red-shifted $A_{1 g}$ peak position, whereas they are anticorrelated with the intensity of the $A_{1 g}$ mode, perhaps owing to the increased sulfur vacancies at local defect regions.

\section{Exciton emission modulated by charge transfer}

\subsection{Near-field imaging of local charge distribution}

Carrier density is a critical factor that determines the PL spectral weights of the exciton species, as well as the overall PL intensity. Near-field imaging techniques provided nanoscale optical imaging of carrier density profiles of 2D-TMDs [34, 79]. Figure 4a displays near-field PL images of ratio of trion emission to exciton emission of $1 \mathrm{~L}-\mathrm{WS}_{2}$ at 6 and $20 \mathrm{~mW}$ laser power. Figure $4 \mathrm{~b}$ shows the representative PL spectra obtained at 6 and $20 \mathrm{~mW}$ laser power.
Figure $4 \mathrm{a}$ and $\mathrm{b}$ both indicate that as the laser power was increased, trion spectral weight increased. Because the energy difference between exciton and trion energy peak represents the trion dissociation energy $\left(E_{\mathrm{ds}}\right)$ that is the sum of the trion binding energy and the Fermi level $\left(E_{\mathrm{F}}\right)$ as schematically depicted in Figure 4c. Thus, the map of $E_{\mathrm{ds}}$ would represent the local distribution of Fermi level $\left(E_{\mathrm{F}}\right)$ assuming the constant value of trion binding energy (estimated to be $\sim 20 \mathrm{meV}$ [80]) or the amount of accumulated charges. Figure $4 \mathrm{~d}$ shows near-field images of $E_{\mathrm{ds}}$ at $6 \mathrm{mV}$ and $20 \mathrm{~mW}$. At $20 \mathrm{~mW}$ laser power, the regions of high $E_{\mathrm{F}}$ are spatially correlated with the line defects, which suggest the local charge population around the line defects. Figure 4e displays near-field PL images of $1 \mathrm{~L}-\mathrm{MoS}_{2}$ obtained at 1.83 and $1.87 \mathrm{eV}$ corresponding to trion (upper side of Figure $3 \mathrm{~b}$ ) and exciton emission (lower side of Figure 3b), respectively [56, 57]. In both images, PL is lower along the line defects but more distinctively in exciton emission, which means the ratio of trion to exciton is higher along the line defects. This observation suggests that the electron density is high at defect sites and grain edges, which tend to be populated with sulfur vacancies $[50,58,81,82]$.

\subsection{Charge exchanges in TMD heterostructures}

Near field imaging can capture the spatial and spectral changes of PL emission caused by the charge transfer and a
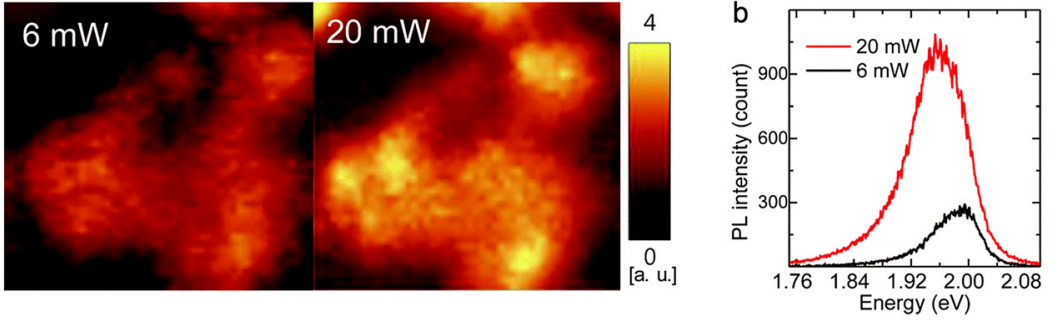

C
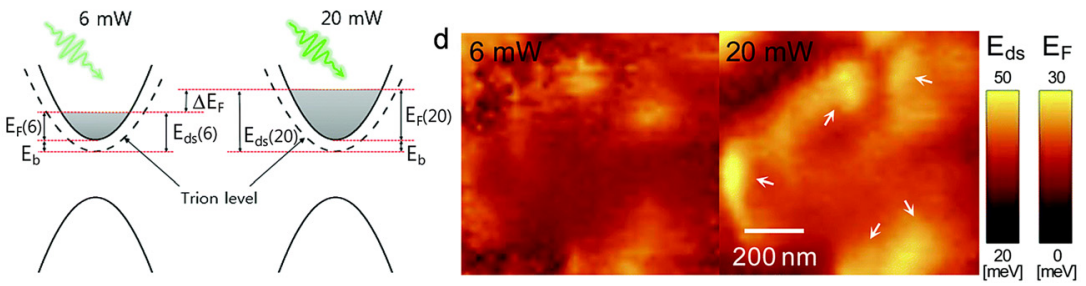

e
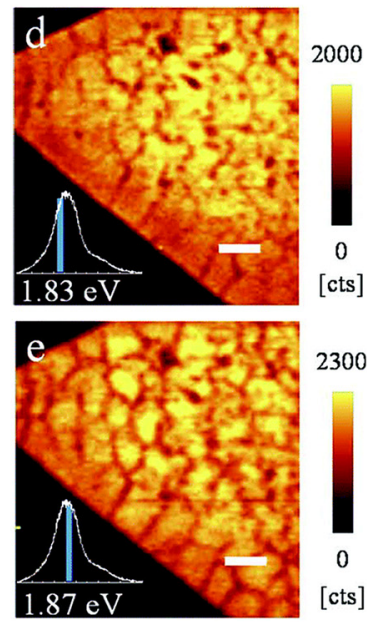

Figure 4: Local charge population of $1 \mathrm{~L}-\mathrm{WS}_{2}$.

(a) Near-field image of the ratio of trion emission to exciton emission at 6 and $20 \mathrm{~mW}$, respectively. (b) Representative near-field PL spectra. (c) Schematic energy band diagram to explain the relation between trion dissociation energy $\left(E_{\mathrm{ds}}\right)$, trion binding energy $\left(E_{\mathrm{b}}\right)$ and Fermi level $\left(E_{\mathrm{f}}\right)$. (d) Near-field images of $E_{\mathrm{ds}}$ or the estimated $E_{\mathrm{F}}$ assuming $E_{\mathrm{b}}$ of $20 \mathrm{meV}$ at 6 and $20 \mathrm{~mW}$. Reproduced with permission from ref. [34]. Copyright 2018, Royal Society of Chemistry. (e) Near-field PL images of trion and exciton of $1 \mathrm{~L}-\mathrm{MoS}_{2}$, respectively. Reproduced with permission from ref. [79]. Copyright 2018, Royal Society of Chemistry. 
exchanges. 1L-TMDs can be vertically stacked to form a vertical heterostructure owing to the weak van der Waals interaction between 2D-TMD layers. Generally, 2D-TMD heterostructures often form a type II band alignment, facilitating the separation of electrons and holes; further, they can host the interlayer exciton, which has a long lifetime [83-85]. The unique characteristics of the heterostructure have been widely utilized in optoelectronic, photodetector, and light-harvesting applications [86-88]. However, in the case of vertical TMD heterostructures, we found only two papers using near-field imaging techniques, probably because of the low intensity of the heterostructure PL due to charge transfer across the heterostructure interface $[89,90]$. TEPL imaging of a vertical $\mathrm{WSe}_{2} / \mathrm{MoSe}_{2}$ heterostructure [89] revealed the intralayer and interlayer exciton dynamics (further discussion on this work is given later in the 5.2 plasmon resonance section). In another study, TEPL imaging of vertical heterostructures of $\mathrm{WSe}_{2} / \mathrm{MoS}_{2}$ and $\mathrm{MoS}_{2} / \mathrm{WSe}_{2}$ [90]. In this study, the blisters and nanobubbles showed different PL signals (more discussion is given in Section 5.1). On the other hand, lateral heterojunctions (HJs) between different kinds of 1L-TMDs have been fabricated, where two domains of different TMDs form a lateral $\mathrm{HJ}$ with atomically sharp interfaces [91-93]. Because physical properties such as the formation of interface width and optical properties such as exciton behavior at the interface are of key interest for application in optoelectronic devices [94, 95], it is important to optically investigate lateral $\mathrm{HJ}$ formation with nanoscale spatial resolution.

Figure 5 shows the results of the near-field PL imaging of lateral $\mathrm{MoSe}_{2} / \mathrm{WSe}_{2} \mathrm{HJ}$ [96-98]. Figure 5a shows the TEPL imaging of the multijunction lateral $\mathrm{HJ}$ of $\mathrm{MoSe}_{2} /$ $\mathrm{WSe}_{2}$ synthesized by a water-assisted CVD growth process $[99,100]$. For the TEPL measurements, they used an Aucoated Ag tip, and the spatial resolution was estimated to be $\sim 40 \mathrm{~nm}$. In this image, the bright and dark regions correspond to $\mathrm{WSe}_{2}$ and $\mathrm{MoSe}_{2}$, respectively. Interestingly, two types of smooth $(\sim 230 \mathrm{~nm})$ and sharp $(\sim 40 \mathrm{~nm})$ interfaces existed, as shown in the inset of Figure 4a, which were caused by the gas switching during the CVD growth [99, 101]. Figure $5 \mathrm{~b}$ also displays the TEPL image of the lateral $\mathrm{HJ}$ of $\mathrm{MoSe}_{2} / \mathrm{WSe}_{2}$ grown by CVD under variable temperature conditions [96]. The interface width of $\mathrm{HJ}$ was $\sim 150 \mathrm{~nm}$, and they further investigated the varying plasmonic effects of the interface region while adjusting the tipsample distance, which is discussed in detail in Section 5.2. Near-field PL imaging using an aperture-type probe with an aperture size of $\sim 90 \mathrm{~nm}$ was also utilized for the investigation of lateral HJ, as shown in Figures $4 \mathrm{~d}$ and $5 \mathrm{c}$ [98]. PL quenching at the interface was observed with a spatial width of $\sim 370 \mathrm{~nm}$, which originated from the exciton charge separation due to the type II band alignment between the $\mathrm{MoSe}_{2}$ and $\mathrm{WSe}_{2}$ monolayers [102]. For the quantitative analysis, a plot of the trion/exciton ratio versus lateral distance across the interface was provided. While approaching the interface, the trion/exciton ratio of $\mathrm{MoSe}_{2}$, which represents the density of excess charge, increased, while that of $\mathrm{WSe}_{2}$ decreased. These results directly indicate that exciton separation occurs at the interface of the lateral $\mathrm{HJ}$.

Several groups have focused on the role of aging effects that result in exciton suppression in lateral TMD HJs. Figure 6 a shows the TEPL images obtained after 2 weeks of growth, where the nanoparticles were generated due to the aging effect [97]. No nanoparticles were observed in the fresh samples, as shown in Figure 5a. Aging-induced nanoparticles prevented the tip-induced enhancement of the near-field PL signal and quenched the PL intensity. In addition, the PL peak position of the nanoparticles exhibited a 2-4 nm red-shift because of the strain at the localized nanoparticle sites. Another group also performed TEPL imaging of $\mathrm{MoS}_{2} / \mathrm{WS}_{2} \mathrm{HJ}$, as shown in Figure $6 \mathrm{~b}$ [103]. The heterostructure consists of $\mathrm{WS}_{2}$ in the outer region and $\mathrm{MoS}_{2}$ in the inner region, forming an $\mathrm{HJ}$ width of $\sim 100 \mathrm{~nm}$. Figure $6 \mathrm{~b}$ shows near-field PL images obtained at $1.94 \mathrm{eV}$ ( $\mathrm{WS}_{2}$ excitons) and $1.81\left(\mathrm{MoS}_{2}\right.$ excitons) with time ranges of 1-103 days. The $\mathrm{WS}_{2}$ and $\mathrm{MoS}_{2}$ regions displayed different degradations of PL intensity. The PL intensity of $\mathrm{WS}_{2}$ showed spatially inhomogeneous degradation, the rate of which rapidly decreased after a few days. By contrast, the $\mathrm{MoS}_{2}$ regions maintained almost the same PL intensity for up to 103 days. This distinct difference could be explained by the fact that the density of sulfur vacancies, which leads to degradation by oxidation, is higher along the edge regions than in the inner regions [104]. In addition, the HJ interface may have blocked oxidation propagating from the edge to the inner regions. Because the formation of $\mathrm{Mo}_{x} \mathrm{~W}_{1-x} \mathrm{~S}_{2}$ alloy is known to be thermodynamically stable [105], the presence of $\mathrm{HJ}$ may have prevented oxidation of the inner region.

\subsection{Effects of chemical doping and passivation}

Chemical doping and defect passivation can modulate the carrier density of 1L-TMDs. Chemical doping by molecular adsorption has been extensively used to transfer charges to 1L-TMDs. For example, the PL intensity of $n$-type 1L-TMDs, such as $\mathrm{MoS}_{2}, \mathrm{MoSe}_{2}$, and $\mathrm{WS}_{2}$, was significantly enhanced by chemical p-doping, which was attributed to the 
a

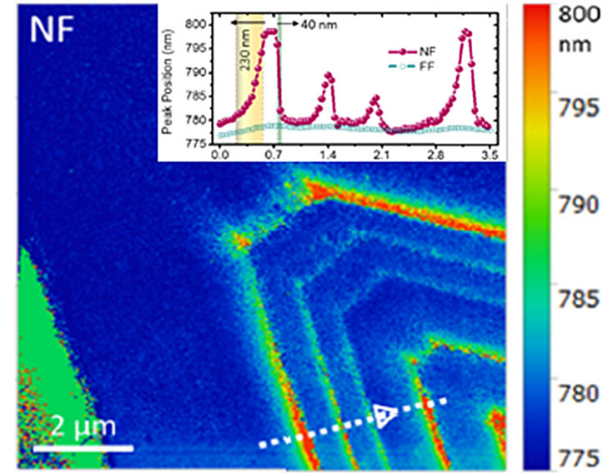

c

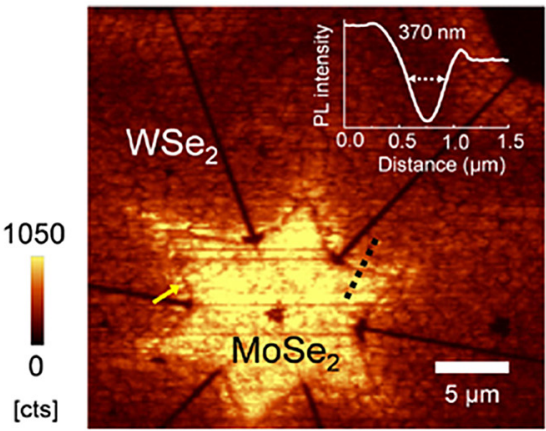

b

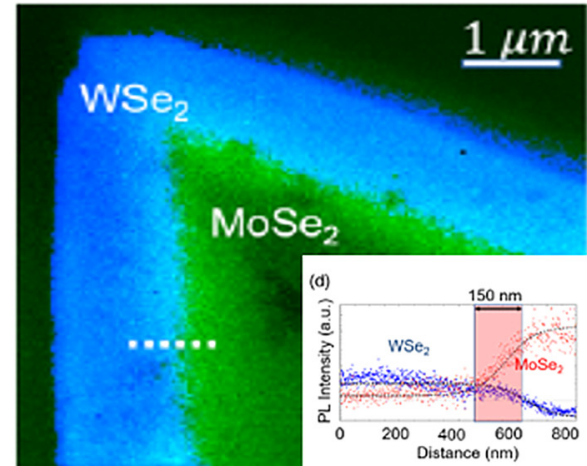

d

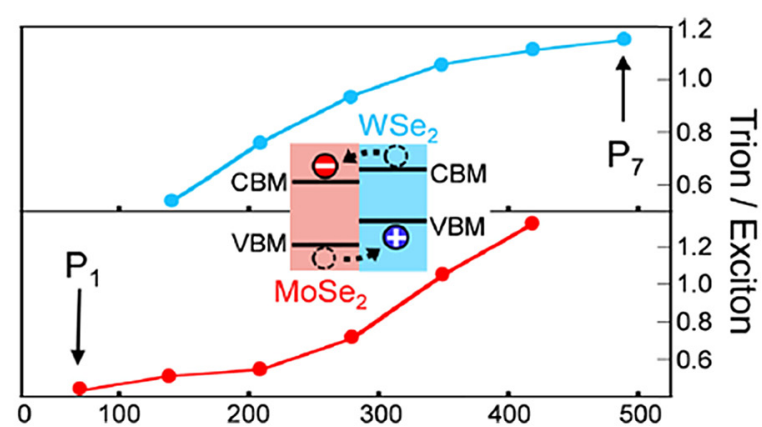

Figure 5: Near-field images of lateral $\mathrm{HJ}$ of $\mathrm{WSe}_{2} / \mathrm{MoSe}_{2}$.

(a) TEPL image. Inset: the line profile obtained along the white dash lines in the TEPL image. Reproduced with permission from ref. [97]. Copyright 2019, Optical Society of America. (b) TEPL image. Inset: the line profile highlighted white dashed line in TEPL image. Reproduced with permission from ref. [96]. Copyright 2018, American Physical Society. (c) Near-field PL image. Inset: the line profile highlighted black dash in near-field PL image. (d) Plot of the trion/exciton ratio of $\mathrm{WSe}_{2}$ (upper) and $\mathrm{MoSe}_{2}$ (lower). Inset: the band alignment of $\mathrm{MoSe}_{2} / \mathrm{WSe}_{2}$ lateral HJ. Reproduced with permission from ref. [98]. Copyright 2019, Optical Society of America.

a
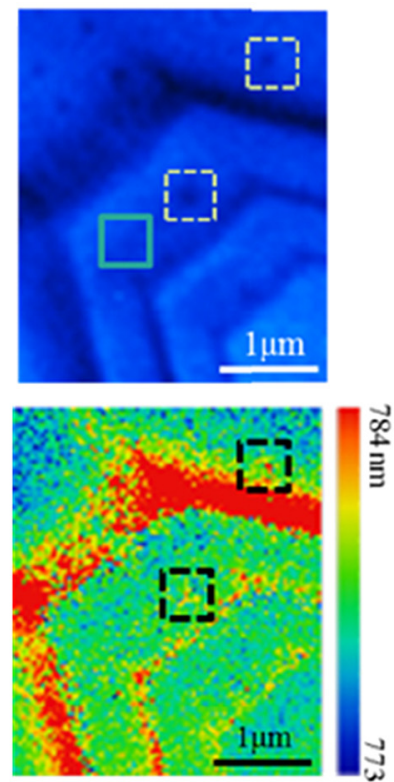

b Day 1
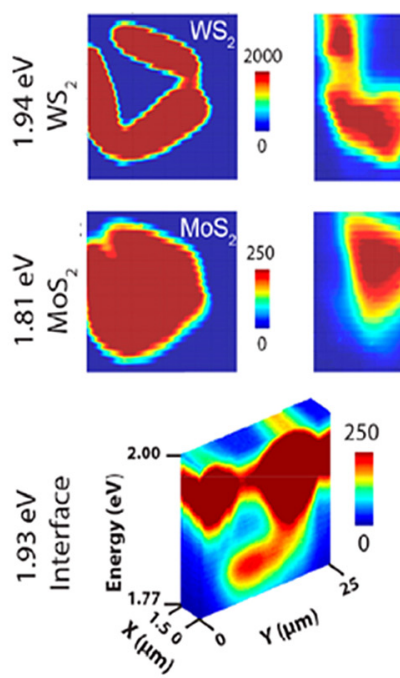

Dav 5
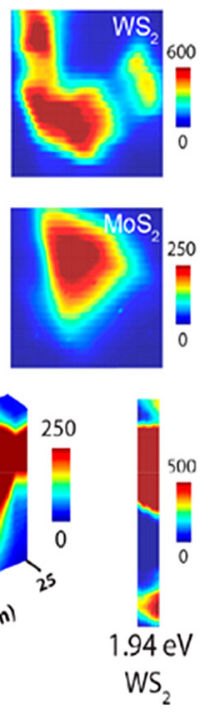

Day 20
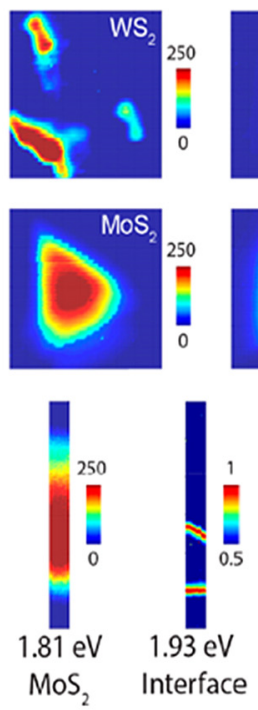

Day 85
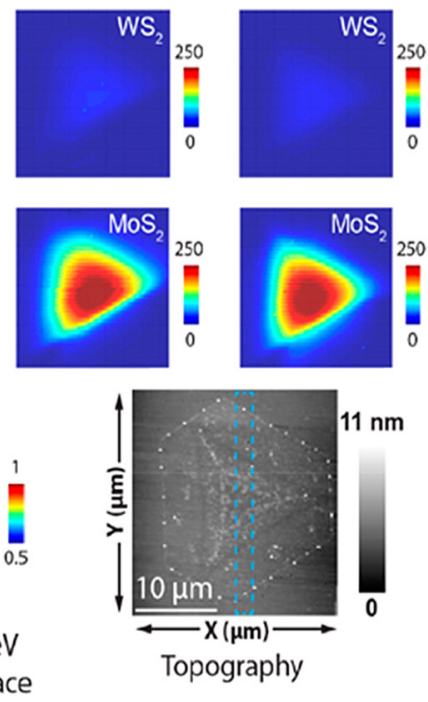

Figure 6: TEPL images that show the aging effect of TMD HJ (a) TEPL intensity (upper panel) and peak position (lower panel) images. Squares display the location of nanoparticles formed by aging effect. Reproduced with permission from ref. [97]. Copyright 2019, Optical Society of America. (b) TEPL images obtained at $1.94 \mathrm{eV}$ (upper) and $1.81 \mathrm{eV}$ (center) and interface (lower). Reproduced with permission from ref. [103]. Copyright 2021, American Chemical Society. 
reduction of charge screening and Auger recombination $[19,57]$. On the other hand, several studies have reported that local defects in 1L-TMDs can be repaired by chemical treatment, greatly increasing the PL emission. Amani et al. showed that exfoliated $1 \mathrm{~L}-\mathrm{MoS}_{2}$ and $1 \mathrm{~L}-\mathrm{WS}_{2}$ treated with bis(trifluoromethane)-sulfonimide (TFSI) showed nearunity QYs [106]; however, mechanism of the PL modulation by chemical treatment is not clear. This is because chemical treatments tend to have concurrent effects on both doping and defect repair. Therefore, in order to understand the exact mechanism, a precise investigation that can spatially and spectrally distinguish the defect and clean regions of $1 \mathrm{~L}-\mathrm{TMD}$ is required. In this section, we discuss the near-field images obtained before and after chemical treatment of $1 \mathrm{~L}-\mathrm{MoS}_{2}$.

Figure 7 displays near-field images of CVD-grown $1 \mathrm{~L}-\mathrm{MoS}_{2}$ obtained before and after TFSI and 7,7,8,8-te tracyanoquinodimethane (TCNQ) that acted as chemical doping [79]. Before chemical treatment, exciton emission was weak at line defects, while trion was relatively strong. This effect more clearly appeared in the trion/total emission ratio image $\left(A^{-} / A^{\text {total }}\right)$. CVD-grown $1 \mathrm{~L}-\mathrm{MoS}_{2}$ was known to be $n$-type and trions are populated at line defects, as shown in Figure 4e, which are in good agreement with previous results [50, 58, 81, 82]. After chemical treatment, total PL emission increased and the exciton and trion emission also followed the trend of total emission. However, the trion/total emission ratio was reduced. This result indicates that the electron depletion by TFSI is more distinct at line defects. On the other hand, in the case of chemical doping by TCNQ, the PL was enhanced in the defect-free region, as shown in Figure $7 \mathrm{~b}$. This means the p-doping by TCNQ was conducted uniformly, whereas electron depletion or p-doping effects by TFSI was prominent at the defect sites.

\section{Effects of strain and plasmon resonances}

\subsection{Strain}

Strain engineering of 2D-TMDs can modulate their electronic, optical, and excitonic properties. For example, exciton funneling of $1 \mathrm{~L}-\mathrm{WS}_{2}$ can be controlled by the

a
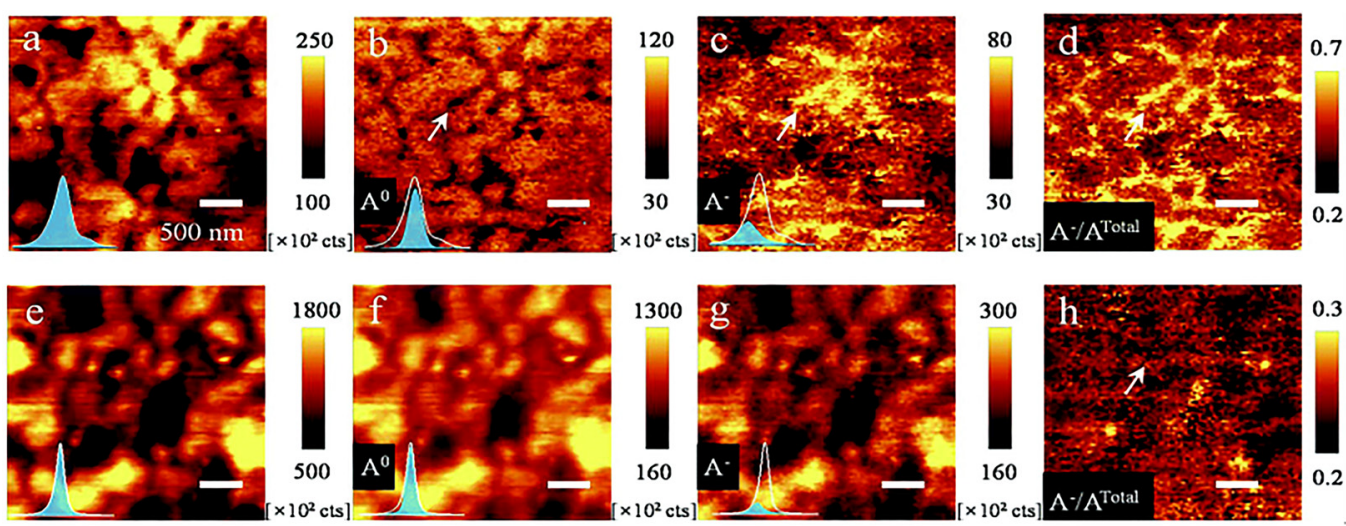

b
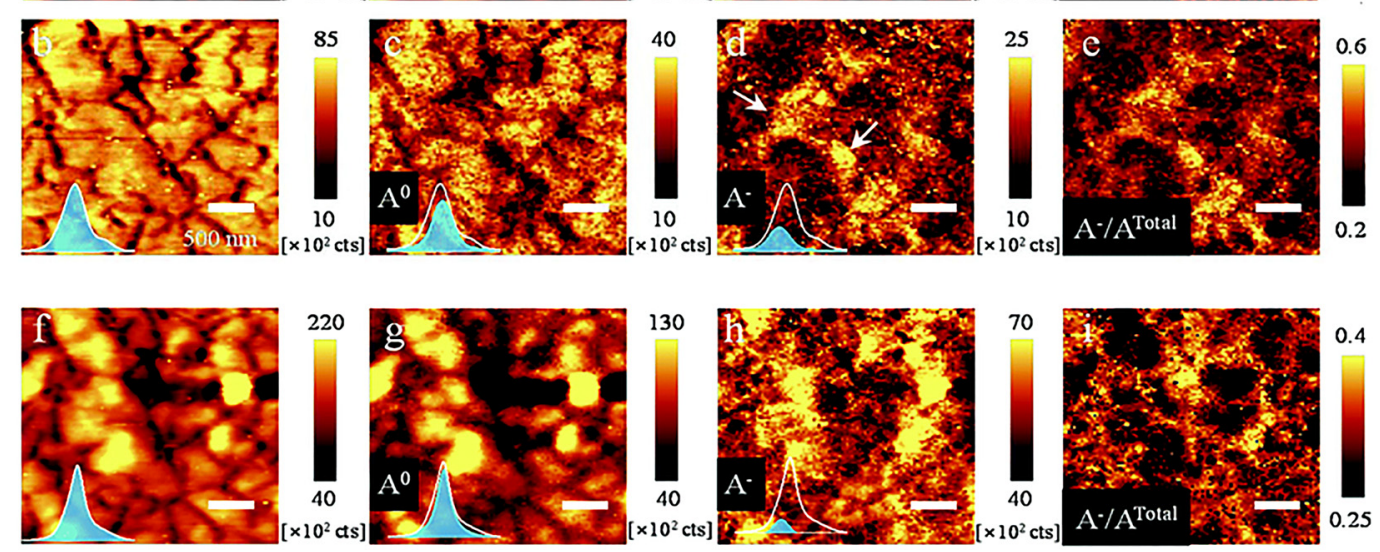

Figure 7: Near-field PL images of 1L-MoS2 by two different chemical treatment.

(a) Near-field PL images of total, exciton, trion, and trion/total ratio 1L-MoS2 before (upper panel) and after TFSI-treatment (lower panel). (b) Near-field PL images of total, exciton, trion, and trion/total ratio of 1L-MoS2 before (upper panel) and after TCNQ-treatment (lower panel). Reproduced with permission from ref. [79]. Copyright 2018, Royal Society of Chemistry. 
formation of topographic wrinkles [107], and the excitonphonon coupling of 1L-TMDs can be modulated by mechanical strain [108], and the band gap can be tuned by the strain gradient in bilayer $\mathrm{MoS}_{2}$ [109]. Strain on 2D-TMDs can be present on bubbles, blisters, and wrinkles that are naturally formed during the growth or transfer process [90, 110] or artificially made by substrate deformation, thermal expansion, and AFM tip contact [107, 111, 112]. Both natural and artificial structures can produce switchable and tunable optical properties of 2D-TMD [113-115]. However, because the strain in 2D-TMDs made by the abovementioned methods is locally generated at the nanoscale, strain effects can hardly be directly observed with far-field optical microscopy. In this section, we discuss how strain effects can affect 2D-TMD, as revealed by near-field imaging.

Bubbles and wrinkles generated by the growth or transfer process can induce strain on 2D-TMDs [90, 110]. Near-field studies on wrinkles were performed using TERS and TEPL, as shown in Figure $8[76,116]$. Figure 8a shows the TERS image of the wrinkles with a spatial resolution of $\sim 20 \mathrm{~nm}$. Wrinkles were well identified by AFM and showed strong TERS intensity. The authors explained that the increase in the TERS signal at the wrinkle is due to the folding of the layers. TEPL imaging of wrinkles was also performed by Rodriguez et al., as shown in Figure 8b [116]. Large and small wrinkles are observed in the TEPL intensity and energy maps in Figure 8b. These different sizes of wrinkles showed different energy peak shifts, which were redshifted at large wrinkles and blue-shifted at small wrinkles. These phenomena can be explained by the fact that large wrinkles combine the effects of energy funneling and the tensile strain effect [117], while small wrinkles result in a compressive strain effect [118]. Another investigation on wrinkles was performed by Koo et al. [35]. They performed TEPL measurements near the wrinkle, which showed the variation of different shapes and intensities, as shown in Figure 8c. At the apex of the wrinkle, the TEPL intensity was stronger, and the peak position was more red-shifted than that at the ground regions. The spectra from the slope regions showed different intensities depending on the distance to the apex or the ground of the wrinkle. They performed TEPL imaging near the wrinkles, as shown in Figure $8 \mathrm{~d}$. From the TEPL and AFM images and as indicated by the dashed line in the TEPL and AFM images, it was found that the peak position was red-shifted and linewidths were reduced in the apex region due to tensile strain, and the region between the slope and the ground showed a lower PL intensity and increased linewidths. This result can be explained by exciton funneling, where the excitons tend to move toward the lower bandgap region. Therefore, the results obtained from the TEPL images were explained by the concurrent effects of tensile strain and funneling [117].

Nanobubbles generated by the transfer process have also been studied using near-field optical techniques. Darlington et al. performed near-field imaging of nanobubbles of $1 \mathrm{~L}-\mathrm{WSe}_{2}$ with $\sim 34 \mathrm{~nm}$ spatial resolution [119]. The localized exciton state emission $(\sim 1.56 \mathrm{eV})$ within the nanobubbles was observed by spatial mapping of the local exciton intensity, and the corresponding AFM image is shown in Figure 9a. In general, the low-energy emission of 2D-TMDs occurs in the case of thermal broadening [120] and plasmonic coupling with the tip [121]. Recently, the observation of localized exciton emission in the nanobubble region of $1 \mathrm{~L}-\mathrm{WSe}_{2}$ was attributed to a single-photon a
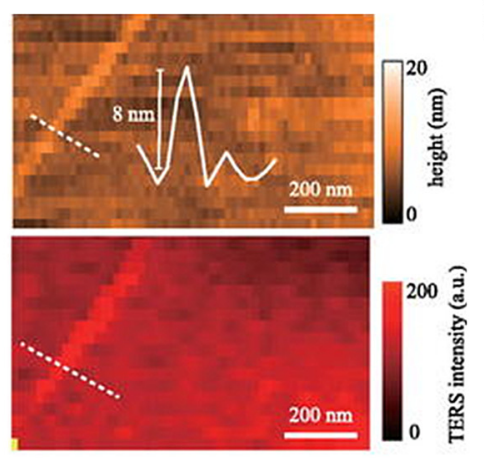

b
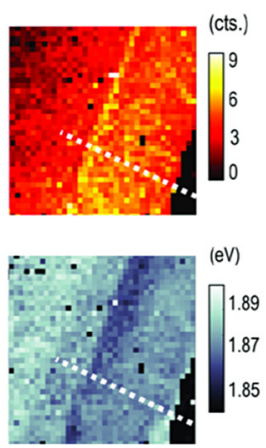

C

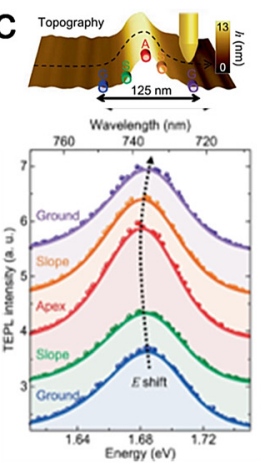

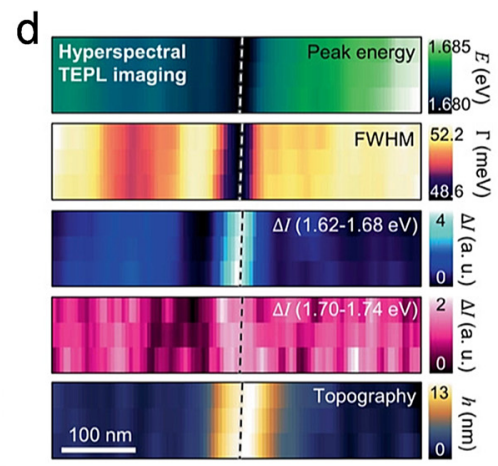

Figure 8: Near-field optical images of wrinkles in 1L-TMDs.

(a) AFM image (upper panel) and TERS intensity image (lower panel) of 1L-MoS 2 . Reproduced with permission from ref. [76]. Copyright 2019, American Institute of Physics. (b) TEPL intensity image (upper panel) and peak energy image (lower panel) of $1 \mathrm{~L}-\mathrm{MoS}_{2}$. Reproduced with permission from ref. [116]. Copyright 2019, WILEY-VCH. (c) Topography of a wrinkle in WSe $e_{2}$ (upper panel) and TEPL spectra obtained at wrinkle (lower panel). (d) TEPL images of peak energy, FWHM, intensity obtained at 1.62-1.68 eV and 1.70-1.74 eV, and topography image.

Reproduced with permission from ref. [35]. Copyright 2021, WILEY-VCH. 
emitter state coupled to a plasmonic cavity at low temperature $[110,122]$. Therefore, the strong low-energy emission originating from the localized state in the nanobubbles was induced due to enhancement by the plasmonic tip. To further analyze the exciton characteristics of nanobubbles, AFM images and corresponding TEPL images of the nanobubbles were obtained, as shown in Figure $9 \mathrm{~b}$. The spatial distribution of the low-energy state exhibited a doughnut-like pattern, and the peak energy was the lowest at the nanobubble periphery, and the PL intensity was strong in the center region. This can be explained by the exciton funneling effect occurring at the central region of the nanobubbles [123], whereas the PL variation at the periphery was dominated by the strain effect. The TEPL investigation of nanobubbles and blisters of vertical TMD heterostructures (i.e., $\mathrm{WSe}_{2} / \mathrm{MoS}_{2}$ on h-BN) was performed by Rodriguez et al. [90]. In their work, the blisters and nanobubbles of $\mathrm{WSe}_{2} / \mathrm{MoS}_{2}$ were identified by TEPL imaging, as shown in Figure 9c. The strain in the blister and nanobubbles was estimated to be 0.5 and $0.9 \%$ by TEPL measurements, respectively. Interestingly, they found that the strain effects of nanobubbles changed depending on the vertical order of the two layers in the heterostructure. Figure $9 \mathrm{~d}$ shows the TEPL mapping images of nanobubbles in vertical $\mathrm{WSe}_{2} / \mathrm{MoS}_{2}$ and $\mathrm{MoS}_{2} / \mathrm{WSe}_{2}$ heterostructures. When $\mathrm{WSe}_{2}$ was placed on top, the PL of only $\mathrm{WSe}_{2}$ was redshifted, as shown in Figure 9c, and the
PL of $\mathrm{MoS}_{2}$ was stronger in the surrounding region of the nanobubble. When $\mathrm{MoS}_{2}$ was placed on top, both layers exhibited PL red-shift due to the strain in the center of the nanobubble, as shown in the spectra in Figure 9d.

We can also control the local strain by artificially manipulating the surface of $1 \mathrm{~L}-\mathrm{TMDs}$. One simple method is to use an AFM tip to apply the contact force to the sample. Park et al. controlled the local strain by adjusting the contact force between the tip and sample [36]. Figure 10a shows the TEPL spectra of $1 \mathrm{~L}-\mathrm{WSe}_{2}$ obtained in an irreversible and reversible state under tip-sample force interaction. The tensile strain in the transferred $1 \mathrm{~L}-\mathrm{WSe}_{2}$ was estimated to be $\sim 0.98 \%$ [124]. When the contact force was sufficiently large, the TEPL intensity increased, and the spectral shift was blue-shifted by $\sim 48 \mathrm{meV}$. Eventually, the strain relaxation becomes irreversible. By contrast, a small force corresponding to a blue shift of $\sim 24 \mathrm{meV}$ was able to reversibly release the strain. This study revealed that the strain can be manipulated by adjusting the tip-sample contact force. The other method was to fabricate a metal-patterned substrate [125]. AFM imaging and corresponding TERS imaging of exfoliated $\mathrm{MoS}_{2}$ transferred onto $\mathrm{Au}$ nanotriangles were performed by Rahaman et al., as shown in Figure 10b. The Raman signal was strong at the corner of the $\mathrm{Au}$ triangle owing to the plasmonic enhancement between the $\mathrm{Au}$ nanotriangles and the Au tip. To quantitatively analyze the strain distribution of $\mathrm{MoS}_{2}$ on the Au substrate, they a
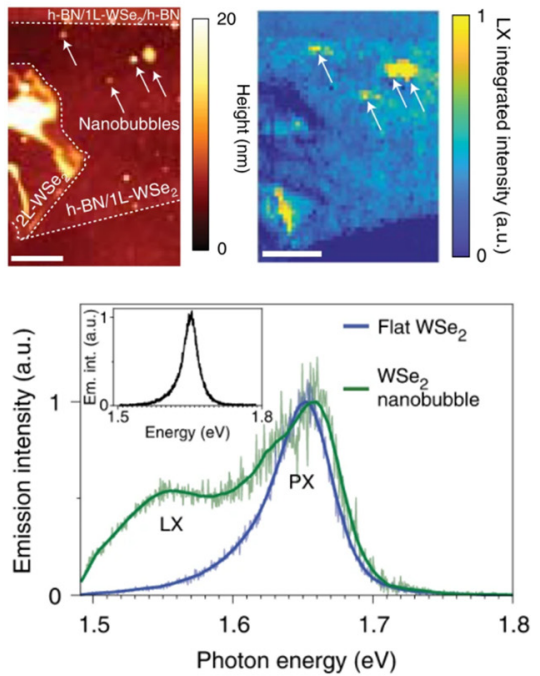
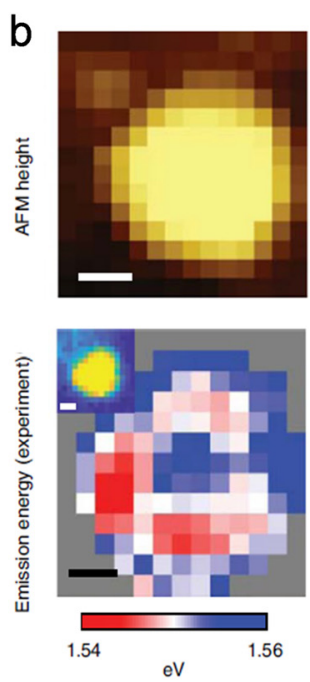
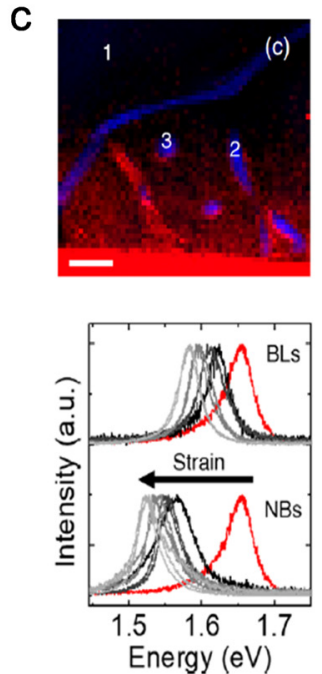
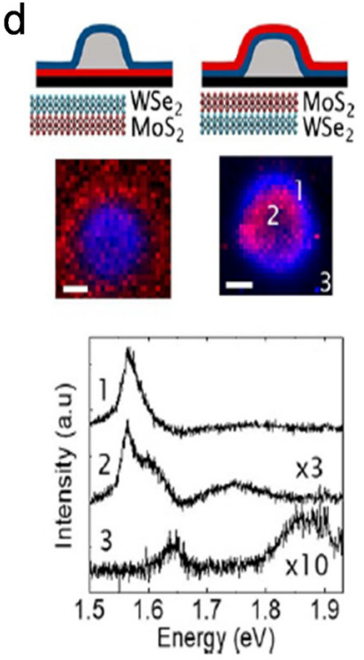

Figure 9: Near-field images of bubbles in 2D-TMDs.

(a) Upper panel: AFM and TEPL images of $1 \mathrm{~L}-\mathrm{WSe}_{2}$. Lower panel: representative TEPL spectra obtained at flat (blue) and nanobubble (green) regions. Inset: far-field spectrum of $1 \mathrm{~L}-\mathrm{WSe}_{2}$. (b) AFM (upper panel) and TEPL peak energy (lower panel) images of a nanobubble. Reproduced with permission from ref. [119]. Copyright 2020, Nature Publishing Group. (c) Upper panel: TEPL image includes flat (\#1), blister (\#2), and nanobubble (\#3) regions of vertical $\mathrm{WSe}_{2} / \mathrm{MoS}_{2}$ heterostructure. Lower panel: TEPL spectra of blisters and nanobubbles. (d) Upper panel: TEPL intensity maps of nanobubbles in (left panel) $\mathrm{WSe}_{2} / \mathrm{MoS}_{2}$ and (right panel) $\mathrm{MoS}_{2} / \mathrm{WSe}_{2}$ heterostructures. Lowe panel: TEPL spectra of the $\mathrm{MoS}_{2} / \mathrm{WSe}_{2}$ nanobubble obtained from TEPL image of $\mathrm{MoS}_{2} / \mathrm{WSe}_{2}$. Reproduced with permission from ref. [90]. Copyright 2021, IOP Publishing. 

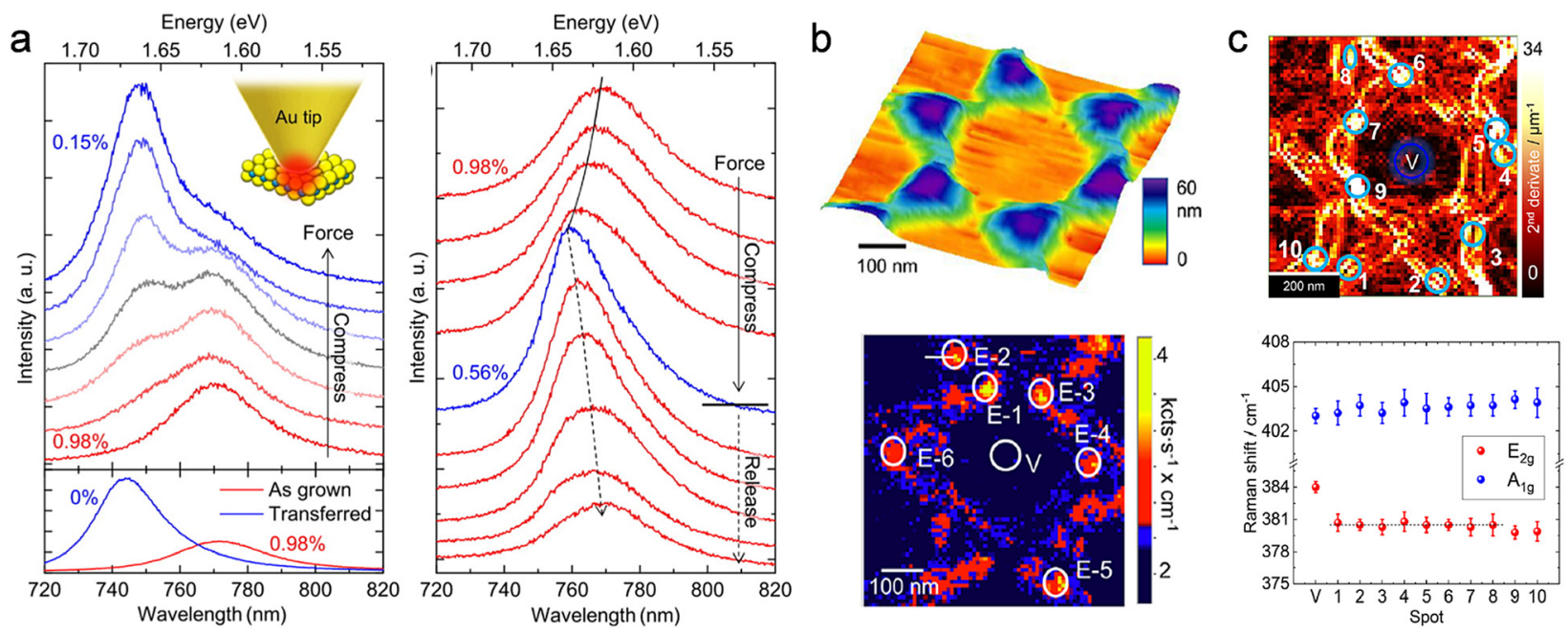

Figure 10: Strain controlled by artificial methods.

(a) Left panel: TEPL spectra of $1 \mathrm{~L}-\mathrm{WSe}_{2}$ with increasing compressive force induced by the tip (upper panel). For comparison, as-grown and transferred 1L-WSe $e_{2}$ is presented (lower panel). Right panel: reversibly controllable TEPL spectra under control of the tip force. Reproduced with permission from ref. [36]. Copyright 2016, Nature Publishing Group. (b) AFM topography (upper) and TERS image (lower) (c) second order derivative image of AFM image (upper) and Raman peak position at the numbered locations indicated by circles (lower). Reproduced with permission from ref. [125]. Copyright.

obtained the second derivative of the AFM image and displayed the Raman shift corresponding to all 11 different locations selected from the AFM image, as shown in Figure 10c. The bright regions are believed to originate from the high curvature formed owing to the relatively larger strain. Because the Raman shifts of $\mathrm{A}_{1 \mathrm{~g}}$ and $\mathrm{E}_{2 \mathrm{~g}}$ are related to doping and strain, respectively [126, 127], no noticeable change in the $A_{1 g}$ peak position indicates a negligible strain, whereas a large shift of the $\mathrm{E}_{2 \mathrm{~g}}$ mode by $4.2 \mathrm{~cm}^{-1}$ compared to the flat surface indicates $\sim 1.4 \%$ strain [127].

\subsection{Plasmon resonance}

Plasmonic effects can be utilized to control the light matter interaction and to enhance the electric field and lightharvesting efficiency $[128,129]$. Thus, plasmonic structures combined with 2D-TMDs can not only enhance their respective optical properties, but also create new ones. For example, plasmonic hot electrons in 2D-TMDs can modulate the carrier density [130], and the coupling between excitons and plasmonic nanocavity can greatly enhance the PL of 2D-TMDs [131, 132]. Measurements with high spatial resolution are advantageous for investigating plasmonic effects. In this section, we focus on near-field plasmon effects studied by near-field imaging.

Figure 11a and $\mathrm{b}$ presents gap-plasmon TERS imaging of $1 \mathrm{~L}-\mathrm{MoS}_{2}$ placed on a periodic array of Au nanostructures [133]. In general, when excited with $785.3 \mathrm{~nm}$, the $\mathrm{A}_{1 \mathrm{~g}}$ mode is suppressed because the $A_{1 g}$ mode can only be excited via an electron-phonon exchange [134]. However, under the same excitation wavelength, the $\mathrm{A}_{1 \mathrm{~g}}$ mode was remarkably enhanced and the $E_{2 g}$ mode vanished, as shown in Figure 11c. This is because the strong plasmonic coupling between $\mathrm{MoS}_{2}$ and the $\mathrm{Au}$ nanostructure can easily generate hot electrons, leading to strong electron-phonon coupling [52, 135], or the perpendicular orientation between the $\mathrm{Au}$ tip and the sample surface enhanced the outof-plane mode $\left(A_{1 g}\right)$ [136]. Additionally, the $A_{2 u}$ mode, referred to as the infrared-active mode, appeared because of the electric field gradient effect occurring between the angled tip and the metal substrate [137]. Furthermore, the Raman spectra obtained by TERS in Figure 11b show a strong signal due to the heating effect, the plasmonic enhancement, and the red-shifted peak position due to the strain effect at the edges of the Au nanocluster. In addition, the observation of the Raman mode at $335 \mathrm{~cm}^{-1}$, characteristic of the 1T phase of $\mathrm{MoS}_{2}$, strongly indicates that the phase transition from $2 \mathrm{H}$ to the $1 \mathrm{~T}$ phase may have occurred at the edge site because the injected hot electrons made the $2 \mathrm{H}$ lattice unstable [52].

On the other hand, many researchers have focused on the effects of the plasmonic mode in the subnanometer gap $(d<\sim 0.5 \mathrm{~nm})$ between the tip and 2D-TMD surface. In general, tip-enhanced signals are known to enhance the electric field by localized surface plasmon resonances using $\mathrm{Au}$ or Ag-coated nanotips at certain distances $(1<d<20 \mathrm{~nm})$ from the sample surface. By contrast, under the van der Waals 
a
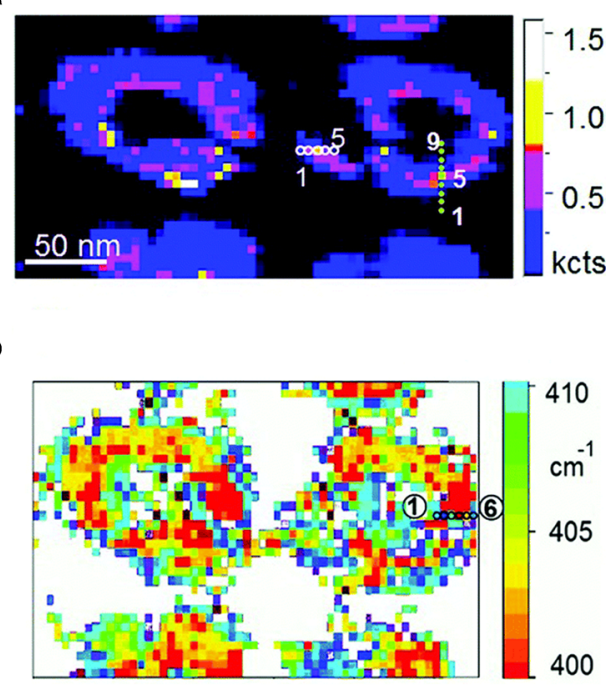

C

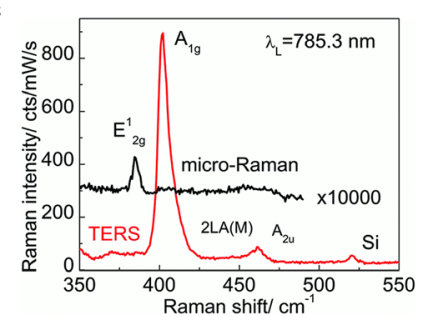

d

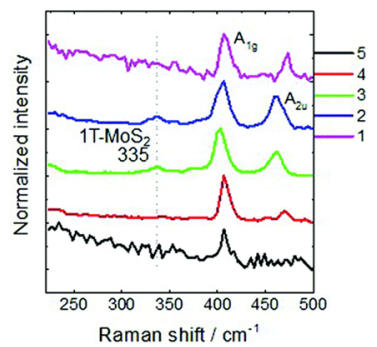

Figure 11: Plasmon resonance effect of $1 \mathrm{~L}-\mathrm{MoS}_{2}$ on an Au nanocluster array. TERS images of intensity (a) and peak position (b). (c) Representative TERS spectrum (red) and far-field spectrum (black). (d) TERS spectra obtained at (b) locations, 1 to 5 marked by a white circle in (b). Reproduced with permission from ref. [133]. Copyright 2018, Royal Society of Chemistry.
(vdW) contact, where $d<\sim 0.5 \mathrm{~nm}$, the quantum plasmonic effect of the charge tunneling between the tip and the sample surface tends to suppress the near-field enhancement [138-140]. Zhang et al. first demonstrated the TERS of few-layer $\mathrm{MoS}_{2}$ on a gold substrate under vdW contact between the tip and sample $(d<\sim 0.36 \mathrm{~nm})$, called the quantum coupling region, as shown in Figure 12a [141]. At a tipsample distance of $2<d<10 \mathrm{~nm}$, which is called the classic coupling region, the PL and Raman signals increase owing to the near-field enhancement. By contrast, in the quantum region, both the PL and Raman signals were saturated at a certain distance and then gradually decreased. Here, the authors explained that the quenched signal was due to a decrease in the electric field in the gap, which occurred because the tunneling between the Au tip and $\mathrm{MoS}_{2}$ reduced the charge accumulation at the apex of the tip.

$\mathrm{He}$ et al. performed TEPL imaging of $1 \mathrm{~L}-\mathrm{WS}_{2}$ by approaching the tip to sample the vdW distance [142]. Figure 12b displays the exciton and trion spectral images in the far-field and near-field. Far-field images of excitons and trions showed that excitons and trions have similar distributions of excitons and trions, while near-field profile images a

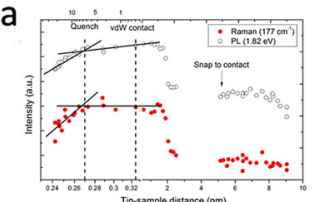

b

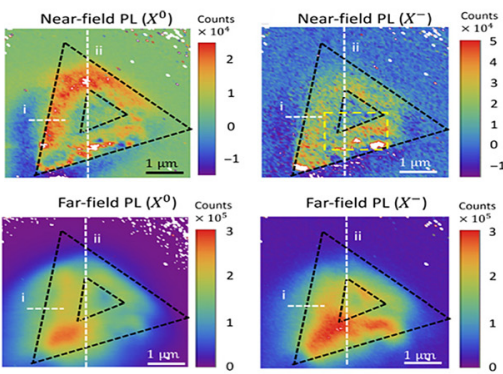

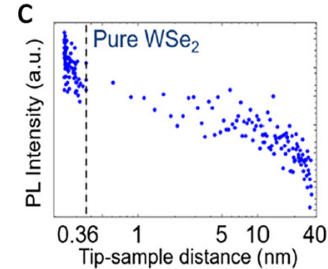

Wavelength $(\mathrm{nm})$

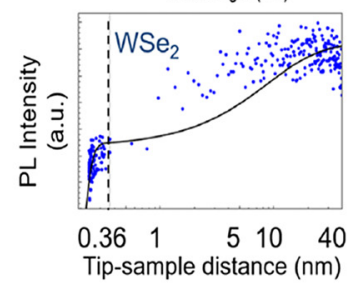

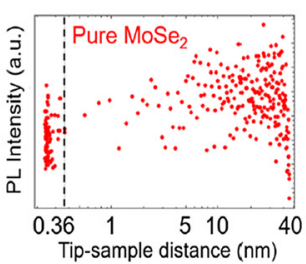

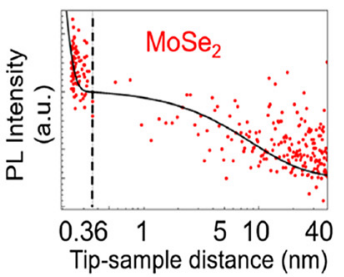

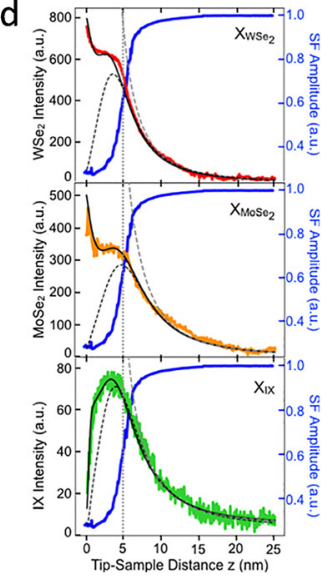

Figure 12: Plasmon resonance effect by metallic tip.

(a) Few-layer $\mathrm{MoS}_{2}$ PL and Raman intensity depending on tip-sample distance (left) and the illustration of tip-induced plasmon resonance (right). Reproduced with permission from ref. [141]. Copyright 2016, Nature Publishing Group. (b) Upper panel: TEPL images of $1 \mathrm{~L}-$ WS $\mathrm{S}_{2}$ of exciton (left) and trion (right). Lower panel: exciton (left) and trion (right) images obtained by far-field imaging. Reproduced with permission from ref. [142]. Copyright 2019, American Association for the Advancement of Science. (c) Upper panel: TEPL intensity of WSe ${ }_{2}$ (left) and MoSe ${ }_{2}$ (right) depending on tip-sample distance. Lower panel: TEPL intensity of lateral WSe $\mathrm{W}_{2} / \mathrm{MoSe}_{2} \mathrm{HJ}_{\text {in depletion region of WSe }}$ (left) and MoSe $\mathrm{C}_{2}$ (right) depending on tip-sample distance. Reproduced with permission from ref. [96]. Copyright 2018, American Physical Society. (d) Fitted TEPL intensity profiles $\mathrm{WSe}_{2}$ (red), $\mathrm{MoSe}_{2}$ (gold), and interlayer exciton peaks (green) versus tip-sample distance obtained from vertical WSe ${ }_{2} /$ $\mathrm{MoSe}_{2}$. Blue lines display the shear-force feedback amplitude. Reproduced with permission from ref. [89]. Copyright 2021, Nature Publishing Group. 
have different distributions because the tip-induced quantum plasmonic effect can convert excitons to trions in certain spatial locations. The interconversion of excitons into trions could be a useful application for novel nanoscale lightmatter interactions. Tang et al. also focused on the tip-sample distance in the depletion region of the lateral $\mathrm{WSe}_{2} / \mathrm{MoSe}_{2}$ heterostructure, as shown in Figure 5b [96]. In the quantum coupling region, the TEPL intensity of pure $\mathrm{WSe}_{2}$ increased with decreasing tip-sample distance, whereas in the case of $\mathrm{MoSe}_{2}$, the PL intensity showed no significant change, as shown in the upper panel of Figure 12c. Because the CVD-grown $\mathrm{WSe}_{2}$ exhibited p-type behavior, which means that the hole concentration in $\mathrm{WSe}_{2}$ was larger than that of electrons, hot electrons injected into $\mathrm{WSe}_{2}$ by the tunneling effect can combine with holes of $\mathrm{WSe}_{2}$ within the vdW distance, leading to PL enhancement [143]. By contrast, CVD-grown $\mathrm{MoSe}_{2}$, n-type, which lacks holes that can couple with the hot electrons, showed no enhancement of the PL signal [144]. However, this trend shows the opposite behavior in the depletion region, as shown in the lower panel of Figure 12c. The hot-electron accumulation in the $\mathrm{WSe}_{2}$ region of $\mathrm{HJ}$ was suppressed owing to the type-II band alignment. Charge transfer across the depletion region caused the overall PL intensity of the $\mathrm{WSe}_{2}$ to be quenched $[84,85]$.

By contrast, the PL signal of $\mathrm{MoSe}_{2}$ increased in the depletion region, and the authors explained that an increase in the number of hot electrons in $\mathrm{MoSe}_{2}$ due to tunneling and charge transfer may increase the recombination rate. However, the Purcell effect by nanocavity may have contributed to this. More recently, May et al. performed TEPL measurements of a vertical $\mathrm{WSe}_{2} / \mathrm{MoSe}_{2}$ heterostructure on h-BN, as shown in Figure 12d [89]. TEPL signals also showed different behaviors depending on the tip-sample distance. The near-field region $(5<d<25 \mathrm{~nm}$ ) showed a continuous increase in the PL signal, regardless of the exciton type. In the $(1<d<5 \mathrm{~nm})$ region, two effects of PL intensity increase due to nearfield enhancement and the decrease due to quantum tunneling competing with each other. In the $d<1 \mathrm{~nm}$ region, owing to the competing effects of the Purcell effect and quantum tunneling, the authors observed the enhancement of intralayer PL and a decrease in the interlayer excitons of the vertical $\mathrm{WSe}_{2} / \mathrm{MoSe}_{2}$ heterostructure. Interestingly, the TEPL of $\mathrm{WSe}_{2}$ in the lateral (Figure 12c) or vertical (Figure 12d) heterostructures showed opposite behaviors in the quantum region. Therefore, a study of the quantum plasmonic region between the tip and the sample still needs to be conducted to understand the integrated plasmonic effects.

Park et al. also observed the dark exciton of $1 \mathrm{~L}-\mathrm{WSe}_{2}$ using TEPL spectroscopy in a room temperature [28]. In general, the dark excitons generated from the spin-flip

Table 1: Summary of experimental details of the near-field optical studies of 2D-TMDs.

\begin{tabular}{|c|c|c|c|c|}
\hline Target of the study & \multicolumn{2}{|l|}{ Sample type } & Probe type & Optical response \\
\hline \multirow[t]{2}{*}{ Grain boundaries } & \multicolumn{2}{|l|}{$1 \mathrm{~L}-\mathrm{MoS}_{2}$} & Apertured [26], scattering type [55], [62] & PL, Raman, SHG \\
\hline & \multicolumn{2}{|l|}{$1 \mathrm{~L}-\mathrm{MoSe}_{2}$} & Scattering type [60], [61] & PL, Raman \\
\hline \multirow[t]{2}{*}{ Local defects } & \multicolumn{2}{|l|}{$1 \mathrm{~L}-\mathrm{MoS}_{2}$} & Apertured [79] & $\mathrm{PL}$ \\
\hline & \multicolumn{2}{|l|}{$1 \mathrm{~L}-\mathrm{WS}_{2}$} & Apertured [34], scattering type [27] & PL, Raman \\
\hline \multirow[t]{3}{*}{ Heterostructures } & Vertically stacked & $\begin{array}{l}\mathrm{WSe} \mathrm{e}_{2} / \mathrm{MoSe}_{2} \\
\mathrm{WSe}_{2} / \mathrm{MoS}_{2}\end{array}$ & $\begin{array}{l}\text { Scattering type }[89] \\
\text { Scattering type }[90]\end{array}$ & \multirow[t]{3}{*}{$\mathrm{PL}$} \\
\hline & \multirow[t]{2}{*}{ Lateral junction } & $\mathrm{WSe}_{2} / \mathrm{MoSe}_{2}$ & Apertured $[98]$, scattering type $[96,97]$ & \\
\hline & & $\mathrm{MoS}_{2} / \mathrm{WS}_{2}$ & Scattering type [103] & \\
\hline Effect of chemical doping and passivation & $1 \mathrm{~L}-\mathrm{MoS}_{2}$ & & Apertured [79] & $\mathrm{PL}$ \\
\hline \multirow[t]{6}{*}{ Strain } & \multirow[t]{2}{*}{ Wrinkles } & $1 \mathrm{~L}-\mathrm{MoS}_{2}$ & Scattering type $[76,116]$ & PL, Raman \\
\hline & & $1 \mathrm{~L}-\mathrm{WSe}_{2}$ & Scattering type $[35]$ & $\mathrm{PL}$ \\
\hline & \multirow[t]{2}{*}{ Bubbles } & $1 \mathrm{~L}-\mathrm{WSe}_{2}$ & Scattering type [119] & PL \\
\hline & & $\begin{array}{l}\text { Vertically stacked } \\
\left(\mathrm{WSe}_{2} / \mathrm{MoS}_{2}\right)\end{array}$ & Scattering type [90] & $\mathrm{PL}$ \\
\hline & AFM tip contact & $1 \mathrm{~L}-\mathrm{WSe}_{2}$ & Scattering type [36] & PL \\
\hline & Au nanotriangle & $1 \mathrm{~L}-\mathrm{MoS}_{2}$ & Scattering type [125] & Raman \\
\hline \multirow[t]{6}{*}{ Plasmon resonance } & \multicolumn{2}{|l|}{$1 \mathrm{~L}-\mathrm{MoS}_{2}$} & Scattering type [133] & Raman \\
\hline & \multicolumn{2}{|l|}{ Few-layer $\mathrm{MoS}_{2}$} & Scattering type [141] & PL, Raman \\
\hline & \multicolumn{2}{|c|}{$1 \mathrm{~L}-\mathrm{WS}_{2}$} & Scattering type [142] & $\mathrm{PL}$ \\
\hline & \multicolumn{2}{|c|}{ Lateral junction $\left(\mathrm{WSe}_{2} / \mathrm{MoSe}_{2}\right)$} & Scattering type [96] & PL \\
\hline & \multicolumn{2}{|c|}{ Vertically stacked $\left(\mathrm{WSe}_{2} / \mathrm{MoSe}_{2}\right)$} & Scattering type [89] & $\mathrm{PL}$ \\
\hline & \multicolumn{2}{|c|}{$1 \mathrm{~L}-\mathrm{WSe} \mathrm{e}_{2}$} & Scattering type [28] & $\mathrm{PL}$ \\
\hline
\end{tabular}


a

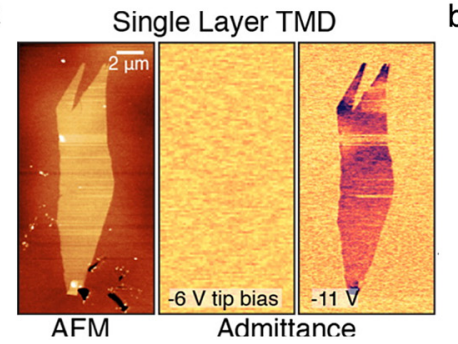

$b$

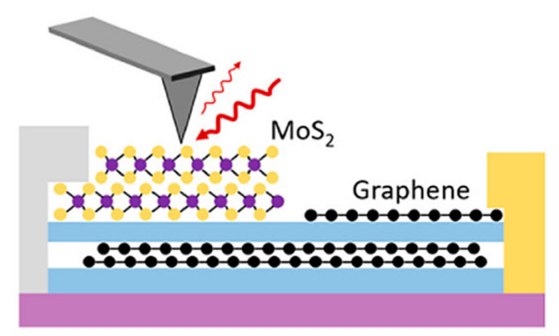

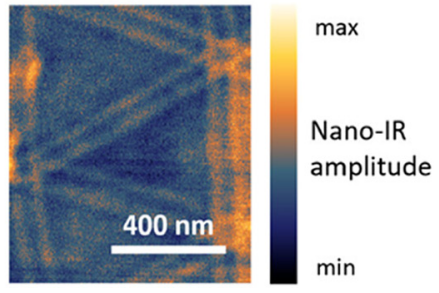

Figure 13: Applications of near-field imaging of 2D-TMDs.

(a) AFM image and microwave near-field images of $1 \mathrm{~L}-\mathrm{MoS}_{2}$ with $-6 \mathrm{~V}$ and $-11 \mathrm{~V}$ tip bias. Reproduced with permission from ref. [150]. Copyright 2015, Nature Publishing Group. (b) Schematic of $\mathrm{MoS}_{2}$-gated 1L-graphene device (left) and near-field-infrared amplitude image (right). Reproduced with permission from ref. [153]. Copyright 2021, Nature Publishing Group.

process cannot be normally observed [145, 146]. Recently, observation of dark excitons was conducted using an inplane magnetic field [147], SPP coupling [148], and polarized detection from the sample edge [149], which requires measurements at low temperatures. Therefore, to meet the demands for selective excitation and out-of-plane transition dipole moments, researchers constructed the required nearfield system. The authors observed that the emission of dark exciton from $1 \mathrm{~L}-\mathrm{WSe}_{2}$ changed with the distance between the $\mathrm{Au}$ tip and the sample. The intensity of out-of-plane optical field gets stronger than that of the in-plane mode at very near distance. Thus, the portion of neutral exciton having in-plane dipole moment decreased as the distance decreased while the emission of dark exciton with out-ofplane dipole moment increased at near distance [147-149]. Therefore, the emissions of dark excitons versus, neutral excitons can be controlled by adjusting the tip-sample distance. Finally, we summarize the experimental details of near-field optical studies of 2D-TMDs in Table1.

\section{Outlook}

To date, near-field imaging has provided information about optical responses and allows for the precise investigation of 2D-TMDs. However, near-field imaging techniques require specific environments regarding substrates, tips, and temperatures to obtain a high spatial resolution. Nevertheless, if near-field imaging can be combined with other measurement systems, it would be of great help to understand the unique 2D-TMD properties and their applications. One possibility is the use of a different light source, as shown in Figure 3e.

Berweger et al. performed microwave near-field imaging of $1 \mathrm{~L}-\mathrm{WSe}_{2}$, as shown in Figure 13a [150]. This work demonstrated the ways to measure electrical properties such as conductivity and capacitance [151,152]. The charge carrier density of $\mathrm{WSe}_{2}$ can be controlled by adjusting the tip bias. In addition, Sunku et al. performed near-field imaging of graphene-based heterostructures using an infrared light source, as shown in Figure 13b [153]. Nearfield imaging showed a pattern showing the propagation of plasmons. In this work, bilayer $\mathrm{MoS}_{2}$ was used as the topgate metal. Although $\mathrm{MoS}_{2}$ exhibited a weaker efficiency of top-gate displacement than monolayer graphene, it is meaningful in that they first performed near-field IR imaging of 2D-TMDs.

Moiré patterns in TMD heterostructures have recently become a popular topic. Although we have already covered the near-field studies of heterostructures of 2D-TMD interlayer excitons, the moiré exciton states depending on stack angles remain an interesting subject that can be investigated by near-field imaging. Theoretical studies have shown that moiré potentials in TMD heterostructures can have a significant effect on optical properties [154]. Many researchers have experimentally observed moiré exciton states in $\mathrm{WSe}_{2} /$ $\mathrm{WS}_{2}$ heterostructures and observed intervalley excitons in $\mathrm{MoSe}_{2} / \mathrm{WSe}_{2}$ heterostructures at low temperatures $[155,156]$. Although the interlayer twist offers an additional degree of freedom to regulate moire excitons, the study of the interlayer exciton dynamics of TMD heterostructures remains a challenging and attractive topic. We expect that a near-field imaging system with $\sim 2 \mathrm{~nm}$ spatial resolution can optically identify the moire pattern. In addition, the valley polarization of 2D-TMD is a unique property of 2D-TMDs that could open new applications [157-159]. Many studies have already investigated valley effects on 2D-TMDs using circular polarization $[160,161]$. However, there have been no near-field studies of valley polarization of 2D-TMDs that require experimental environments such as low temperature, proper excitation sources, and magnetic fields. We expect that valley-resolved near-field optical imaging will provide critical information for the further development of 2D-TMD valleytronics. 
Author contributions: All the authors have accepted responsibility for the entire content of this submitted manuscript and approved submission.

Research funding: JK acknowledges the Basic Science Research Programs through the National Research Foundation of Korea (NRF) funded by the Ministry of Education (2019R1A2C1006586; 2021R1A6A1A03039696).

Conflict of interest statement: The authors declare no conflicts of interest regarding this article.

\section{References}

[1] K. S. Novoselov, A. K. Geim, S. V. Morozov, et al., "Electric field effect in atomically thin carbon films," Science, vol. 306, pp. 666-669, 2004.

[2] K. I. Bolotin, K. J. Sikes, Z. Jiang, et al., "Ultrahigh electron mobility in suspended graphene," Solid State Commun., vol. 146, pp. 351-355, 2008.

[3] C. Lee, X. Wei, J. W. Kysar, and J. Hone, "Measurement of the elastic properties and intrinsic strength of monolayer graphene," Science, vol. 321, pp. 385-388, 2008.

[4] B. Anasori, Y. Xie, M. Beidaghi, et al., "Two-dimensional, ordered, double transition metals carbides (MXenes)," ACS Nano, vol. 9, pp. 9507-9516, 2015.

[5] A. S. Anir, S. Akhtar, and J. Kim, “Light-emitting MXene quantum dots," Opto-Electron. Adv., vol. 4, p. 200077, 2021.

[6] K. F. Mak, C. Lee, J. Hone, J. Shan, and T. F. Heinz, "Atomically thin $\mathrm{MoS}_{2}$ : a new direct-gap semiconductor," Phys. Rev. Lett., vol. 105, p. 136805, 2010.

[7] A. Chernikov, T. C. Berkelbach, H. M. Hill, et al., "Exciton binding energy and nonhydrogenic Rydberg series in monolayer $\mathrm{WS}_{2}$," Phys. Rev. Lett., vol. 113, p. 076802, 2014.

[8] M. M. Ugeda, A. J. Bradley, S.-F. Shi, et al., "Giant bandgap renormalization and excitonic effects in a monolayer transition metal dichalcogenide semiconductor," Nat. Mater., vol. 13, pp. 1091-1095, 2014.

[9] H. Zeng and X. Cui, "An optical spectroscopic study on twodimensional group-VI transition metal dichalcogenides," Chem. Soc. Rev., vol. 44, pp. 2629-2642, 2015.

[10] Z. Sun, A. Martinez, and F. Wang, "Optical modulators with 2D layered materials,” Nat. Photonics, vol. 10, pp. 227-238, 2015.

[11] G. Wang, A. Chernikov, M. M. Glazov, et al., "Colloquium: excitons in atomically thin transition metal dichalcogenides," Rev. Mod. Phys., vol. 90, p. 021001, 2018.

[12] S. Das, D. Pandey, J. Thomas, and T. Roy, "The role of graphene and other 2D materials in solar photovoltaics," Adv. Mater., vol. 31, p. 1802722, 2019.

[13] H. Li, G. Lu, Z. Yin, et al., "Optical identification of single- and few-layer $\mathrm{MoS}_{2}$ sheets," Small, vol. 8, pp. 682-686, 2012.

[14] R. Addou, L. Colombo, and R. M. Wallace, "Surface defects on natural $\mathrm{MoS}_{2}$," ACS Appl. Mater. Interfaces, vol. 7, pp. 11921-11929, 2015.

[15] L. Fei, S. Lei, and W.-B. Zhang, "Direct TEM observations of growth mechanisms of two-dimensional $\mathrm{MoS}_{2}$ flakes," Nat. Commun., vol. 7, p. 12206, 2016.
[16] N. Gierlinger and M. Schwanninger, "Chemical imaging of poplar wood cell walls by confocal Raman microscopy," Plant Physiol., vol. 140, pp. 1246-1254, 2006.

[17] D. L. Duong, G. H. Han, and S. M. Lee, "Probing graphene grain boundaries with optical microscopy," Nature, vol. 490, pp. 235-239, 2012.

[18] N. Gierlinger, T. Keplinger, and M. Harrington, "Imaging of plant cell walls by confocal Raman microscopy," Nat. Protoc., vol. 7, pp. 1694-1708, 2012.

[19] K. P. Dhakal, D. L. Duong, J. Lee, et al., "Confocal absorption spectral imaging of $\mathrm{MoS}_{2}$ : optical transitions depending on the atomic thickness of intrinsic and chemically doped $\mathrm{MoS}_{2}$," Nanoscale, vol. 6, pp. 13028-13035, 2014.

[20] R. Tabaksblat, R. J. Meier, and B. J. Kip, "Confocal Raman microspectroscopy: theory and application to thin polymer samples," Appl. Spectrosc., vol. 46, pp. 60-68, 1992.

[21] Y. Kim, E. J. Lee, S. Roy, et al., "Measurement of lateral and axial resolution of confocal Raman microscope using dispersed carbon nanotubes and suspended graphene," Curr. Appl. Phys., vol. 20, pp. 71-77, 2020.

[22] R. Beams, L. G. Cançado, A. Jorio, A. N. Vamivakas, and L. Novotny, "Tip-enhanced Raman mapping of local strain in graphene," Nanotechnology, vol. 26, p. 175702, 2015.

[23] H. Groß, J. M. Hamm, T. Tufarelli, O. Hess, and B. Hecht, "Nearfield strong coupling of single quantum dots," Sci. Adv., vol. 4, p. 4906, 2018.

[24] Y.-C. Yong, Y.-Z. Wang, and J.-J. Zhong, "Nano-spectroscopic imaging of proteins with near-field scanning optical microscopy (NSOM)," Curr. Opin. Biotechnol., vol. 54, pp. 106-113, 2018.

[25] S. Parida, A. Patsha, K. K. Madapu, and S. Dhara, "Nanospectroscopic and nanoscopic imaging of single GaN nanowires in the sub-diffraction limit," J. Appl. Phys., vol. 127, p. 173103, 2020.

[26] Y. Lee, S. Park, H. Kim, et al., "Characterization of the structural defects in CVD-grown monolayered $\mathrm{MoS}_{2}$ using near-field photoluminescence imaging," Nanoscale, vol. 7, pp. 11909-11914, 2015.

[27] C. Lee, B. G. Jeong, S. J. Yun, Y. H. Lee, S. M. Lee, and M. S. Jeong, "Unveiling defect-related Raman mode of monolayer $\mathrm{WS}_{2}$ via tip-enhanced resonance Raman scattering," ACS Nano, vol. 12, pp. 9982-9990, 2018.

[28] K.-D. Park, T. Jiang, G. Clark, X. Xu, and M. B. Raschke, "Radiative control of dark excitons at room temperature by nano-optical antenna-tip Purcell effect," Nat. Nanotechnol., vol. 13, pp. 59-64, 2018.

[29] F. Keilmann and R. Hillenbrand, "Near-field microscopy by elastic light scattering from a tip," Philos. Trans. R. Soc. London, Ser. A, vol. 362, pp. 787-805, 2004.

[30] S.-f. Wu, "Review of near-field optical microscopy," Front. Phys. China, vol. 1, pp. 263-274, 2006.

[31] P. Bazylewski, S. Ezugwu, and G. Fanchini, "A review of threedimensional scanning near-field optical microscopy (3D-SNOM) and its applications in nanoscale light management," Appl. Sci., vol. 7, p. 973, 2017.

[32] Y. Li, A. Li, Y. Zhang, et al., "Nanoscale characterization of surface plasmon-coupled photoluminescence enhancement in pseudo micro blue LEDs using near-field scanning optical microscopy," Nanomaterials, vol. 10, p. 751, 2020. 
[33] H. Lee, D. Y. Lee, M. G. Kang, Y. Koo, T. Kim, and K.-D. Park, "Tip-enhanced photoluminescence nano-spectroscopy and nano-imaging," Nanophotonics, vol. 9, pp. 3089-3110, 2020.

[34] Y. Lee, S. J. Yun, Y. Kim, et al., "Near-field spectral mapping of individual exciton complexes of monolayer $\mathrm{WS}_{2}$ correlated with local defects and charge population," Nanoscale, vol. 9, pp. 2272-2278, 2017.

[35] Y. Koo, Y. Kim, S. H. Choi, et al., "Tip-induced nano-engineering of strain, bandgap, and exciton funneling in 2D semiconductors," Adv. Mater., vol. 33, p. 2008234, 2021.

[36] K.-D. Park, O. Khatib, V. Kravtsov, G. Clark, X. Xu, and M. B. Raschke, "Hybrid tip-enhanced nanospectroscopy and nanoimaging of monolayer $\mathrm{WSe}_{2}$ with local strain control," Nano Lett., vol. 16, pp. 2621-2627, 2016.

[37] G. Nehrke and J. Nouet, "Confocal Raman microscope mapping as a tool to describe different mineral and organic phases at high spatial resolution within marine biogenic carbonates: case study on Nerita undata (Gastropoda, Neritopsina)," Biogeosciences, vol. 8, pp. 3761-3769, 2011.

[38] C. Neumann, S. Reichardt, P. Venezuela, et al., "Raman spectroscopy as probe of nanometre-scale strain variations in graphene," Nat. Commun., vol. 6, p. 8429, 2015.

[39] E. H. Synge, "XXXVIII. A suggested method for extending microscopic resolution into the ultra-microscopic region," Philos. Mag. A, vol. 6, pp. 356-362, 1928.

[40] E. A. Ash and G. Nicholls, "Super-resolution aperture scanning microscope,” Nature, vol. 237, pp. 510-512, 1972.

[41] A. Lewis, M. Isaacson, A. Harootunian, and A. Muray, "Development of a $500 \AA$ spatial resolution light microscope: I. light is efficiently transmitted through $\lambda / 16$ diameter apertures," Ultramicroscopy, vol. 13, pp. 227-231, 1984.

[42] D. W. Pohl, W. Denk, and M. Lanz, "Optical stethoscopy: image recording with resolution $\lambda / 20$," Appl. Phys. Lett., vol. 44, pp. 651-653, 1984

[43] E. Betzig, A. Lewis, A. Harootunian, M. Isaacson, and E. Kratschmer, "Near field scanning optical microscopy (NSOM): development and biophysical applications," Biophys. J., vol. 49, pp. 269-279, 1986.

[44] E. Betzig, J. K. Trautman, T. D. Harris, J. S. Weiner, and R. L. Kostelak, "Breaking the diffraction barrier: optical microscopy on a nanometric scale," Science, vol. 251, pp. 1468-1470, 1991.

[45] F. Keilmann, D. W. van der Weide, T. Eickelkamp, D. Merz, and R. Stöckle, "Extreme sub-wavelength resolution with a scanning radio-frequency transmission microscope," Opt. Commun., vol. 129, pp. 15-18, 1996.

[46] X. Duan, C. Wang, A. Pan, R. Yu, and X. Duan, "Two-dimensional transition metal dichalcogenides as atomically thin semiconductors: opportunities and challenges," Chem. Soc. Rev., vol. 44, pp. 8859-8876, 2015.

[47] J. S. Ross, S. Wu, H. Yu, et al., "Electrical control of neutral and charged excitons in a monolayer semiconductor," Nat. Commun., vol. 4, p. 1474, 2013.

[48] M. S. Kim, S. J. Yun, Y. Lee, et al., "Biexciton emission from edges and grain boundaries of triangular $\mathrm{WS}_{2}$ monolayers," ACS Nano, vol. 10, pp. 2399-2405, 2016

[49] C. Robert, B. Han, P. Kapuscinski, et al., "Measurement of the spin-forbidden dark excitons in $\mathrm{MoS}_{2}$ and $\mathrm{MoSe}_{2}$ monolayers," Nat. Commun., vol. 11, p. 4037, 2020.
[50] A. M. van der Zande, P. Y. Huang, D. A. Chenet, et al., "Grains and grain boundaries in highly crystalline monolayer molybdenum disulphide," Nat. Mater., vol. 12, pp. 554-561, 2013.

[51] Z. Liu, M. Amani, S. Najmaei, et al., "Strain and structure heterogeneity in $\mathrm{MoS}_{2}$ atomic layers grown by chemical vapour deposition," Nat. Commun., vol. 5, p. 5246, 2014.

[52] Y. Kang, S. Najmaei, Z. Liu, et al., "Plasmonic hot electron induced structural phase transition in a $\mathrm{MoS}_{2}$ monolayer," Adv. Mater., vol. 26, pp. 6467-6471, 2014

[53] M. O’Brien, N. McEvoy, T. Hallam, et al., "Transition metal dichalcogenide growth via close proximity precursor supply," Sci. Rep., vol. 4, p. 7374, 2014.

[54] S. Cai, W. Zhao, A. Zafar, et al., "Photoluminescence characterization of the grain boundary thermal stability in chemical vapor deposition grown $\mathrm{WS}_{2}$," Mater. Res. Express, vol. 4, p. 106202, 2017.

[55] W. Bao, N. J. Borys, C. Ko, et al., "Visualizing nanoscale excitonic relaxation properties of disordered edges and grain boundaries in monolayer molybdenum disulfide," Nat. Commun., vol. 6, p. 7993, 2015.

[56] S. Tongay, J. Zhou, C. Ataca, et al., "Broad-range modulation of light emission in two-dimensional semiconductors by molecular physisorption gating," Nano Lett., vol. 13, pp. 2831-2836, 2013.

[57] S. Mouri, Y. Miyauchi, and K. Matsuda, "Tunable photoluminescence of monolayer $\mathrm{MoS}_{2}$ via chemical doping," Nano Lett., vol. 13, pp. 5944-5948, 2013.

[58] W. Zhou, X. Zou, S. Najmaei, et al., "Intrinsic structural defects in monolayer molybdenum disulfide," Nano Lett., vol. 13, pp. 2615-2622, 2013.

[59] H. Wang, C. Zhang, and F. Rana, "Ultrafast dynamics of defectassisted electron-hole recombination in monolayer $\mathrm{MoS}_{2}$," Nano Lett., vol. 15, pp. 339-345, 2015.

[60] D. Moore, K. Jo, C. Nguyen, et al., "Uncovering topographically hidden features in $2 \mathrm{D} \mathrm{MoSe}$ with correlated potential and optical nanoprobes," npj 2D Mater. Appl., vol. 4, p. 44, 2020.

[61] J. Pei, J. Yang, X. Wang, et al., "Excited state biexcitons in atomically thin $\mathrm{MoSe}_{2}$," ACS Nano, vol. 11, pp. 7468-7475, 2017.

[62] A. Raja, L. Waldecker, J. Zipfel, et al., "Dielectric disorder in twodimensional materials," Nat. Nanotechnol., vol. 14, pp. 832-837, 2019.

[63] K. K. H. Smithe, A. V. Krayev, C. S. Bailey, et al., "Nanoscale heterogeneities in monolayer $\mathrm{MoSe}_{2}$ revealed by correlated scanning probe microscopy and tip-enhanced Raman spectroscopy," ACS Appl. Nano Mater., vol. 1, pp. 572-579, 2018.

[64] K. Koike, R. Wada, S. Yagi, Y. Harada, S. Sasa, and M. Yano, "Characteristics of $\mathrm{MoO}_{3}$ films grown by molecular beam epitaxy,” Jpn. J. Appl. Phys., vol. 53, p. 05FJ02, 2014.

[65] N. Illyaskutty, S. Sreedhar, G. Sanal Kumar, et al., "Alteration of architecture of $\mathrm{MoO}_{3}$ nanostructures on arbitrary substrates: growth kinetics, spectroscopic and gas sensing properties," Nanoscale, vol. 6, pp. 13882-13894, 2014.

[66] N. Kumar, S. Najmaei, Q. Cui, et al., "Second harmonic microscopy of monolayer $\mathrm{MoS}_{2}$," Phys. Rev. B, vol. 87, p. 161403, 2013

[67] K. L. Seyler, J. R. Schaibley, and P. Gong, "Electrical control of second-harmonic generation in a $\mathrm{WSe}_{2}$ monolayer transistor," Nat. Nanotechnol., vol. 10, pp. 407-411, 2015. 
[68] J. Cheng, T. Jiang, Q. Ji, et al., "Kinetic nature of grain boundary formation in as-grown $\mathrm{MoS}_{2}$ monolayers," Adv. Mater., vol. 27, pp. 4069-4074, 2015.

[69] K.-D. Park and M. B. Raschke, "Polarization control with plasmonic antenna tips: a universal approach to optical nanocrystallography and vector-field imaging," Nano Lett., vol. 18, pp. 2912-2917, 2018.

[70] J. W. Suk, A. Kitt, C. W. Magnuson, et al., "Transfer of CVD-grown monolayer graphene onto arbitrary substrates," ACS Nano, vol. 5, pp. 6916-6924, 2011.

[71] J. Zhao, Q. Deng, T. H. Ly, G. H. Han, G. Sandeep, and M. H. Rümmeli, "Two-dimensional membrane as elastic shell with proof on the folds revealed by three-dimensional atomic mapping," Nat. Commun., vol. 6, p. 8935, 2015.

[72] S. Tongay, J. Suh, C. Ataca, et al., "Defects activated photoluminescence in two-dimensional semiconductors: interplay between bound, charged and free excitons," Sci. Rep., vol. 3, p. 2657, 2013.

[73] T. Kato and T. Kaneko, "Optical detection of a highly localized impurity state in monolayer tungsten disulfide," ACS Nano, vol. 8, pp. 12777-12785, 2014.

[74] D. V. Voronine, G. Lu, D. Zhu, and A. Krayev, "Tip-enhanced Raman scattering of $\mathrm{MoS}_{2}$," IEEE J. Sel. Top. Quant. Electron., vol. 23, pp. 138-143, 2017.

[75] Y. Okuno, O. Lancry, A. Tempez, et al., "Probing the nanoscale light emission properties of a CVD-grown $\mathrm{MoS}_{2}$ monolayer by tip-enhanced photoluminescence," Nanoscale, vol. 10, pp. 14055-14059, 2018.

[76] R. Kato, T. Umakoshi, R. T. Sam, and P. Verma, “Probing nanoscale defects and wrinkles in $\mathrm{MoS}_{2}$ by tip-enhanced Raman spectroscopic imaging," Appl. Phys. Lett., vol. 114, p. $073105,2019$.

[77] A. Molina-Sánchez and L. Wirtz, "Phonons in single-layer and few-layer MoS 2 and WS 2 ," Phys. Rev. B, vol. 84, p. 155413, 2011.

[78] M. Yamamoto, S. T. Wang, and M. Ni, "Strong enhancement of Raman scattering from a bulk-inactive vibrational mode in fewlayer MoTe 2 ," ACS Nano, vol. 8, pp. 3895-3903, 2014.

[79] Y. Kim, Y. Lee, H. Kim, S. Roy, and J. Kim, "Near-field exciton imaging of chemically treated $\mathrm{MoS}_{2}$ monolayers," Nanoscale, vol. 10, pp. 8851-8858, 2018.

[80] K.F. Mak, K. He, C. Lee, et al., "Tightly bound trions in monolayer MoS $_{2}$," Nat. Mater., vol. 12, pp. 207-211, 2013.

[81] S. Najmaei, Z. Liu, and W. Zhou, "Vapour phase growth and grain boundary structure of molybdenum disulphide atomic layers," Nat. Mater., vol. 12, pp. 754-759, 2013.

[82] G. Tai, T. Zeng, J. Yu, et al., "Fast and large-area growth of uniform $\mathrm{MoS}_{2}$ monolayers on molybdenum foils," Nanoscale, vol. 8, pp. 2234-2241, 2016.

[83] S. Ovesen, S. Brem, C. Linderälv, et al., "Interlayer exciton dynamics in van der Waals heterostructures," Commun. Phys., vol. 2, p. 23, 2019.

[84] X. Hong, J. Kim, S.-F. Shi, et al., "Ultrafast charge transfer in atomically thin $\mathrm{MoS}_{2} / \mathrm{WS}_{2}$ heterostructures," Nat. Nanotechnol., vol. 9, pp. 682-686, 2014.

[85] F. Ceballos, M. Z. Bellus, H.-Y. Chiu, and H. Zhao, "Ultrafast charge separation and indirect exciton formation in $\mathrm{a} \mathrm{MoS}_{2}$ $\mathrm{MoSe}_{2}$ van der Waals heterostructure," ACS Nano, vol. 8, pp. 12717-12724, 2014.
[86] M. S. Choi, D. Qu, D. Lee, et al., "Lateral $M_{0} S_{2}$ p-n junction formed by chemical doping for use in high-performance optoelectronics," ACS Nano, vol. 8, pp. 9332-9340, 2014.

[87] Y. Xue, Y. Zhang, Y. Liu, et al., "Scalable production of a fewlayer $\mathrm{MoS}_{2} / \mathrm{WS}_{2}$ vertical heterojunction array and its application for photodetectors," ACS Nano, vol. 10, pp. 573-580, 2016.

[88] Q. Zeng and Z. Liu, "Novel optoelectronic devices: transitionmetal-dichalcogenide-based 2D heterostructures," $A d v$. Electron. Mater., vol. 4, p. 1700335, 2018.

[89] M. A. May, T. Jiang, C. Du, et al., "Nanocavity clock spectroscopy: resolving competing exciton dynamics in $\mathrm{WSe}_{2} /$ $\mathrm{MoSe}_{2}$ heterobilayers," Nano Lett., vol. 21, pp. 522-528, 2021.

[90] A. Rodriguez, M. Kalbáč, and O. Frank, "Strong localization effects in the photoluminescence of transition metal dichalcogenide heterobilayers," 2D Mater., vol. 8, p. 025028, 2021.

[91] Y. Gong, J. Lin, X. Wang, et al., "Vertical and in-plane heterostructures from $\mathrm{WS}_{2} / \mathrm{MoS}_{2}$ monolayers," Nat. Mater., vol. 13, pp. 1135-1142, 2014.

[92] M.-Y. Li, Y. Shi, C.-C. Cheng, et al., "Epitaxial growth of a monolayer $\mathrm{WSe}_{2}-\mathrm{MoS}_{2}$ lateral $\mathrm{p}-\mathrm{n}$ junction with an atomically sharp interface," Science, vol. 349, pp. 524-528, 2015.

[93] F. Ullah, Y. Sim, C. T. Le, et al., "Growth and simultaneous valleys manipulation of two-dimensional $\mathrm{MoSe}_{2}-\mathrm{WSe}_{2}$ lateral heterostructure," ACS Nano, vol. 11, pp. 8822-8829, 2017.

[94] A. Pospischil, M. M. Furchi, and T. Mueller, "Solar-energy conversion and light emission in an atomic monolayer $\mathrm{p}-\mathrm{n}$ diode," Nat. Nanotechnol., vol. 9, pp. 257-261, 2014.

[95] B. W. H. Baugher, H. O. H. Churchill, Y. Yang, and P. JarilloHerrero, "Optoelectronic devices based on electrically tunable p-n diodes in a monolayer dichalcogenide," Nat. Nanotechnol., vol. 9, pp. 262-267, 2014.

[96] C. Tang, Z. He, W. Chen, S. Jia, J. Lou, and D. V. Voronine, "Quantum plasmonic hot-electron injection in lateral WSe $\mathrm{S}_{2}$ $\mathrm{MoSe}_{2}$ heterostructures," Phys. Rev. B, vol. 98, p. 041402 , 2018.

[97] P. K. Sahoo, H. Zong, J. Liu, et al., "Probing nano-heterogeneity and aging effects in lateral $2 \mathrm{D}$ heterostructures using tipenhanced photoluminescence," Opt. Mater. Express, vol. 9, pp. 1620-1631, 2019.

[98] Y. Kim, S. J. Yun, E. Lee, and J. Kim, "Near-field visualization of charge transfer at $\mathrm{MoSe}_{2} / \mathrm{WSe}_{2}$ lateral heterojunction," Opt. Mater. Express, vol. 9, pp. 1864-1871, 2019.

[99] P. K. Sahoo, S. Memaran, Y. Xin, L. Balicas, and H. R. Gutiérrez, "One-pot growth of two-dimensional lateral heterostructures via sequential edge-epitaxy," Nature, vol. 553, pp. 63-67, 2018.

[100] W. Xue, P. K. Sahoo, J. Liu, et al., "Nano-optical imaging of monolayer $\mathrm{MoSe}_{2}-\mathrm{WSe}_{2}$ lateral heterostructure with subwavelength domains," J. Vac. Sci. Technol, A, vol. 36, p. 05G502, 2018.

[101] C. Huang, S. Wu, A. M. Sanchez, et al., ““'Lateral heterojunctions within monolayer $\mathrm{MoSe}_{2}-\mathrm{WSe}_{2}$ semiconductors," Nat. Mater., vol. 13, pp. 1096-1101, 2014.

[102] J. Kang, S. Tongay, J. Zhou, J. Li, and J. Wu, "Band offsets and heterostructures of two-dimensional semiconductors," Appl. Phys. Lett., vol. 102, p. 012111, 2013. 
[103] A. Fali, T. Zhang, J. P. Terry, et al., "Photodegradation protection in $2 \mathrm{D}$ in-plane heterostructures revealed by hyperspectral nanoimaging: the role of nanointerface 2D alloys," ACS Nano, vol. 15, pp. 2447-2457, 2021.

[104] V. Carozo, Y. Wang, K. Fujisawa, et al., "Optical identification of sulfur vacancies: bound excitons at the edges of monolayer tungsten disulfide," Sci. Adv., vol. 3, p. e1602813, 2017.

[105] H.-P. Komsa and A. V. Krasheninnikov, "Two-dimensional transition metal dichalcogenide alloys: stability and electronic properties," J. Phys. Chem. Lett., vol. 3, pp. 3652-3656, 2012.

[106] M. Amani, D.-H. Lien, D. Kiriya, et al., "Near-unity photoluminescence quantum yield in $\mathrm{MoS}_{2}$," Science, vol. 350, pp. 1065-1068, 2015.

[107] J. Lee, S. J. Yun, C. Seo, et al., "Switchable, tunable, and directable exciton funneling in periodically wrinkled $\mathrm{WS}_{2}$," Nano Lett., vol. 21, pp. 43-50, 2021.

[108] I. Niehues, R. Schmidt, M. Drüppel, et al., "Strain control of exciton-phonon coupling in atomically thin semiconductors," Nano Lett., vol. 18, pp. 1751-1757, 2018.

[109] P. Koskinen, I. Fampiou, and A. Ramasubramaniam, "Densityfunctional tight-binding simulations of curvature-controlled layer decoupling and band-gap tuning in bilayer $\mathrm{MoS}_{2}$," Phys. Rev. Lett., vol. 112, p. 186802, 2014.

[110] G. D. Shepard, O. A. Ajayi, X. Li, et al., "Nanobubble induced formation of quantum emitters in monolayer semiconductors," 2D Mater., vol. 4, p. 021019, 2017.

[111] S. Manzeli, A. Allain, A. Ghadimi, and A. Kis, "Piezoresistivity and strain-induced band gap tuning in atomically thin $\mathrm{MoS}_{2}$," Nano Lett., vol. 15, pp. 5330-5335, 2015.

[112] K. Wang, A. A. Puretzky, Z. Hu, et al., "Strain tolerance of twodimensional crystal growth on curved surfaces," Sci. Adv., vol. 5, p. eaav4028, 2019.

[113] W. Wu, L. Wang, Y. Li, et al., "Piezoelectricity of single-atomiclayer $\mathrm{MoS}_{2}$ for energy conversion and piezotronics," Nature, vol. 514, pp. 470-474, 2014.

[114] M. G. Harats, J. N. Kirchhof, M. Qiao, K. Greben, and K. I. Bolotin, "Dynamics and efficient conversion of excitons to trions in nonuniformly strained monolayer $\mathrm{WS}_{2}$," Nat. Photonics, vol. 14, pp. 324-329, 2020.

[115] A. R. Khan, T. Lu, W. Ma, Y. Lu, and Y. Liu, "Tunable optoelectronic properties of WS $\mathrm{W}_{2}$ by local strain engineering and folding," Adv. Electron. Mater., vol. 6, p. 1901381, 2020.

[116] A. Rodriguez, T. Verhagen, M. Kalbac, J. Vejpravova, and 0 . Frank, "Imaging nanoscale inhomogeneities and edge delamination in as-grown $\mathrm{MoS}_{2}$ using tip-enhanced photoluminescence," Phys. Status Solidi RRL, vol. 13, p. 1900381, 2019.

[117] A. Castellanos-Gomez, R. Roldán, E. Cappelluti, et al., “Local strain engineering in atomically thin $\mathrm{MoS}_{2}$," Nano Lett., vol. 13, pp. 5361-5366, 2013.

[118] M. López-Suárez, I. Neri, and R. Rurali, "Band gap engineering of $\mathrm{MoS}_{2}$ upon compression,”J. Appl. Phys., vol. 119, p. 165105, 2016.

[119] T. P. Darlington, C. Carmesin, M. Florian, et al., "Imaging strainlocalized excitons in nanoscale bubbles of monolayer $\mathrm{WSe}_{2}$ at room temperature," Nat. Nanotechnol., vol. 15, pp. 854-860, 2020.
[120] Y. Luo, N. Liu, X. Li, J. C. Hone, and S. Strauf, "Single photon emission in $\mathrm{WSe}_{2}$ up $160 \mathrm{~K}$ by quantum yield control," $2 D$ Mater., vol. 6, p. 035017, 2019.

[121] M. S. Eggleston, K. Messer, L. Zhang, E. Yablonovitch, and M. C. Wu, "Optical antenna enhanced spontaneous emission," Proc. Natl. Acad. Sci. Unit. States Am., vol. 112, pp. 1704-1709, 2015.

[122] J. Kern, I. Niehues, P. Tonndorf, et al., "Nanoscale positioning of single-photon emitters in atomically thin $\mathrm{WSe}_{2}$," Adv. Mater., vol. 28, pp. 7101-7105, 2016.

[123] J. Feng, X. Qian, C.-W. Huang, and J. Li, "Strain-engineered artificial atom as a broad-spectrum solar energy funnel," Nat. Photonics, vol. 6, pp. 866-872, 2012.

[124] S. B. Desai, G. Seol, J. S. Kang, et al., "Strain-induced indirect to direct bandgap transition in multilayer WSe ${ }_{2}$," Nano Lett., vol. 14, pp. 4592-4597, 2014.

[125] M. Rahaman, R. D. Rodriguez, G. Plechinger, et al., "Highly localized strain in a $\mathrm{MoS}_{2} / \mathrm{Au}$ heterostructure revealed by tipenhanced Raman spectroscopy," Nano Lett., vol. 17, pp. 6027-6033, 2017.

[126] B. Chakraborty, A. Bera, D. V. S. Muthu, S. Bhowmick, U. V. Waghmare, and A. K. Sood, "Symmetry-dependent phonon renormalization in monolayer $\mathrm{MoS}_{2}$ transistor," Phys. Rev. B, vol. 85, p. 161403, 2012.

[127] D. Lloyd, X. Liu, J. W. Christopher, et al., "Band gap engineering with ultralarge biaxial strains in suspended monolayer $\operatorname{MoS}_{2}$," Nano Lett., vol. 16, pp. 5836-5841, 2016.

[128] S. F. Shi, T. T. Tang, B. Zeng, et al., "Controlling graphene ultrafast hot carrier response from metal-like to semiconductor-like by electrostatic gating," Nano Lett., vol. 14, pp. 1578-1582, 2014.

[129] S. Zu, Y. Bao, and Z. Fang, "Planar plasmonic chiral nanostructures," Nanoscale, vol. 8, pp. 3900-3905, 2016.

[130] Y. Yu, Z. Ji, S. Zu, et al., "Ultrafast plasmonic hot electron transfer in Au nanoantenna/MoS 2 heterostructures," Adv. Funct. Mater., vol. 26, pp. 6394-6401, 2016.

[131] H. Y. Jeong, U. J. Kim, H. Kim, et al., "Optical gain in $\mathrm{MoS}_{2}$ via coupling with nanostructured substrate: fabry-perot interference and plasmonic excitation," ACS Nano, vol. 10, pp. 8192-8198, 2016

[132] S. Wang, S. Li, T. Chervy, et al., "Coherent coupling of WS2 monolayers with metallic photonic nanostructures at room temperature," Nano Lett., vol. 16, pp. 4368-4374, 2016.

[133] A. G. Milekhin, M. Rahaman, E. E. Rodyakina, A. V. Latyshev, V. M. Dzhagan, and D. R. T. Zahn, "Giant gap-plasmon tipenhanced Raman scattering of $\mathrm{MoS}_{2}$ monolayers on $\mathrm{Au}$ nanocluster arrays," Nanoscale, vol. 10, pp. 2755-2763, 2018.

[134] T. Yang, X. Huang, H. Zhou, G. Wu, and T. Lai, "Excitation mechanism of $A_{1 g}$ mode and origin of nonlinear temperature dependence of Raman shift of CVD-grown mono- and few-layer $\mathrm{MoS}_{2}$ films," Opt. Express, vol. 24, pp. 12281-12292, 2016.

[135] Z. Li, Y. Xiao, and Y. Gong, "Active light control of the $\mathrm{MoS}_{2}$ monolayer exciton binding energy," ACS Nano, vol. 9, pp. 10158-10164, 2015.

[136] C. C. Neacsu, S. Berweger, and M. B. Raschke, "Tip-enhanced Raman imaging and nanospectroscopy: sensitivity, symmetry, and selection rules," NanoBiotechnology, vol. 3, pp. 172-196, 2007. 
[137] L. Meng, Z. Yang, J. Chen, and M. Sun, "Effect of electric field gradient on sub-nanometer spatial resolution of tip-enhanced Raman spectroscopy," Sci. Rep., vol. 5, p. 9240, 2015.

[138] C. Ciracì, R. T. Hill, J. J. Mock, et al., "Probing the ultimate limits of plasmonic enhancement,” Science, vol. 337, pp. 1072-1074, 2012.

[139] J. Mertens, A. L. Eiden, D. O. Sigle, et al., “Controlling subnanometer gaps in plasmonic dimers using graphene," Nano Lett., vol. 13, pp. 5033-5038, 2013.

[140] V. Kravtsov, S. Berweger, J. M. Atkin, and M. B. Raschke, "Control of plasmon emission and dynamics at the transition from classical to quantum coupling," Nano Lett., vol. 14, pp. 5270-5275, 2014.

[141] Y. Zhang, D. V. Voronine, S. Qiu, et al., "Improving resolution in quantum subnanometre-gap tip-enhanced Raman nanoimaging," Sci. Rep., vol. 6, p. 25788, 2016.

[142] Z. He, Z. Han, J. Yuan, et al., "Quantum plasmonic control of trions in a picocavity with monolayer WS 2 ," Sci. Adv., vol. 5, p. eaau8763, 2019.

[143] H. Zhou, C. Wang, J. C. Shaw, et al., "Large area growth and electrical properties of p-type $\mathrm{WSe}_{2}$ atomic layers," Nano Lett., vol. 15, pp. 709-713, 2015.

[144] X. Wang, Y. Gong, G. Shi, et al., "Chemical vapor deposition growth of crystalline monolayer MoSe $\mathrm{M}_{2}$, ACS Nano, vol. 8, pp. 5125-5131, 2014.

[145] X.-X. Zhang, Y. You, S. Y. F. Zhao, and T. F. Heinz, “Experimental evidence for dark excitons in monolayer WSe ${ }_{2}$," Phys. Rev. Lett., vol. 115, p. 257403, 2015.

[146] A. O. Slobodeniuk and D. M. Basko, "Spin-flip processes and radiative decay of dark intravalley excitons in transition metal dichalcogenide monolayers," 2D Mater., vol. 3, p. 035009, 2016.

[147] X.-X. Zhang, T. Cao, Z. Lu, et al., "Magnetic brightening and control of dark excitons in monolayer WSe $\mathrm{W}_{2}, \mathrm{Nat}$. Nanotechnol., vol. 12, pp. 883-888, 2017.

[148] Y. Zhou, G. Scuri, D. S. Wild, et al., "Probing dark excitons in atomically thin semiconductors via near-field coupling to surface plasmon polaritons," Nat. Nanotechnol., vol. 12, pp. 856-860, 2017.

[149] G. Wang, C. Robert, M. M. Glazov, et al., “In-plane propagation of light in transition metal dichalcogenide monolayers: optical selection rules," Phys. Rev. Lett., vol. 119, p. 047401, 2017.
[150] S. Berweger, J. C. Weber, J. John, et al., "Microwave near-field imaging of two-dimensional semiconductors," Nano Lett., vol. 15, pp. 1122-1127, 2015.

[151] H. P. Huber, M. Moertelmaier, T. M. Wallis, et al., "Calibrated nanoscale capacitance measurements using a scanning microwave microscope," Rev. Sci. Instrum., vol. 81, p. 113701, 2010.

[152] K. Lai, M. Nakamura, W. Kundhikanjana, et al., "Mesoscopic percolating resistance network in a strained manganite thin film," Science, vol. 329, pp. 190-193, 2010.

[153] S. S. Sunku, D. Halbertal, R. Engelke, et al., "Dual-gated graphene devices for near-field nano-imaging," Nano Lett., vol. 21, pp. 1688-1693, 2021.

[154] H. Yu, G.-B. Liu, J. Tang, X. Xu, and W. Yao, “Moiré excitons: from programmable quantum emitter arrays to spin-orbit-coupled artificial lattices," Sci. Adv., vol. 3, p. e1701696, 2017.

[155] C. Jin, E. C. Regan, A. Yan, et al., "Observation of moiré excitons in $\mathrm{WSe}_{2} / \mathrm{WS}_{2}$ heterostructure superlattices," Nature, vol. 567, pp. 76-80, 2019.

[156] K. L. Seyler, P. Rivera, H. Yu, et al., "Signatures of moiré-trapped valley excitons in $\mathrm{MoSe}_{2} / \mathrm{WSe}_{2}$ heterobilayers," Nature, vol. 567, pp. 66-70, 2019.

[157] A. Singh, K. Tran, M. Kolarczik, et al., "Long-lived valley polarization of intravalley trions in monolayer $\mathrm{WSe}_{2}$," Phys. Rev. Lett., vol. 117, p. 257402, 2016.

[158] W.-Y. Tong, S.-J. Gong, X. Wan, and C.-G. Duan, "Concepts of ferrovalley material and anomalous valley Hall effect," Nat. Commun., vol. 7, p. 13612, 2016.

[159] M. Onga, Y. Zhang, T. Ideue, and Y. Iwasa, "Exciton Hall effect in monolayer $\mathrm{MoS}_{2}$," Nat. Mater., vol. 16, pp. 1193-1197, 2017.

[160] P. K. Nayak, F.-C. Lin, C.-H. Yeh, J.-S. Huang, and P.-W. Chiu, "Robust room temperature valley polarization in monolayer and bilayer WS 2 ," Nanoscale, vol. 8, pp. 6035-6042, 2016.

[161] J. Xia, X. Wang, B. K. Tay, et al., "Valley polarization in stacked $\mathrm{MoS}_{2}$ induced by circularly polarized light," Nano Res., vol. 10, pp. 1618-1626, 2017.

Supplementary Material: The online version of this article offers supplementary material (https://doi.org/10.1515/nanoph-2021-0383). 